ARTICLE

\title{
An anchoring complex recruits katanin for microtubule severing at the plant cortical nucleation sites
}

\author{
Noriyoshi Yagi (1) 1,2,3, Takehide Kato (1) 1, Sachihiro Matsunaga (i) 2,4, David W. Ehrhardt (10) 5,6, \\ Masayoshi Nakamura (i) ${ }^{3 凶} \&$ Takashi Hashimoto ${ }^{1 凶}$
}

Microtubules are severed by katanin at distinct cellular locations to facilitate reorientation or amplification of dynamic microtubule arrays, but katanin targeting mechanisms are poorly understood. Here we show that a centrosomal microtubule-anchoring complex is used to recruit katanin in acentrosomal plant cells. The conserved protein complex of Msd1 (also known as SSX2IP) and Wdr8 is localized at microtubule nucleation sites along the microtubule lattice in interphase Arabidopsis cells. Katanin is recruited to these sites for efficient release of newly formed daughter microtubules. Our cell biological and genetic studies demonstrate that Msd1-Wdr8 acts as a specific katanin recruitment factor to cortical nucleation sites (but not to microtubule crossover sites) and stabilizes the association of daughter microtubule minus ends to their nucleation sites until they become severed by katanin. Molecular coupling of sequential anchoring and severing events by the evolutionarily conserved complex renders microtubule release under tight control of katanin activity.

\footnotetext{
${ }^{1}$ Division of Biological Science, Nara Institute of Science and Technology, Nara, Japan. ${ }^{2}$ Faculty of Science and Technology, Department of Applied Biological Science, Tokyo University of Science, Chiba, Japan. ${ }^{3}$ Institute of Transformative Bio-Molecules (WPI-ITbM), Nagoya University, Nagoya, Japan.

${ }^{4}$ Department of Integrated Biosciences, Graduate School of Frontier Sciences, University of Tokyo, Chiba, Japan. ${ }^{5}$ Department of Biology, Stanford University, Stanford, CA, USA. ${ }^{6}$ Department of Plant Biology, Carnegie Institution for Science, Stanford, CA, USA. ${ }^{凶}$ email: mnakamu@itbm.nagoya-u.ac.jp; hasimoto@bs.naist.jp
} 
$\mathrm{M}$ icrotubules in eukaryotic cells adopt various organizations, such as radial arrays centered on centrosomes in many animal cell types and transverse cortical arrays seen in anisotropically elongating and non-centrosomal plant cells ${ }^{1}$. Newly formed microtubules are either anchored at or released from the cellular nucleation sites and the control of such anchoring-release contributes to the array organization ${ }^{2,3}$. Mitotic spindle disanchored 1 (Msd1) was initially discovered as a yeast coiled-coil protein that is required to anchor the minus end of spindle microtubules to the spindle-pole body, the centrosome equivalent in fission yeast ${ }^{4}$. The synovial sarcoma $\mathrm{X}$ breakpoint protein (SSX2IP), an animal ortholog of Msd1, is localized to centriolar satellites that surround the centrosome and is essential for microtubule anchoring and centrosome integrity ${ }^{5-7}$. Msd1/ SSX2IP is targeted to the microtubule nucleation sites partly by its interactions with components of the $\gamma$-tubulin ring complex $(\gamma \mathrm{TuRC})^{4,5}$ and forms a functional protein complex with a WD40-repeat protein, Wdr8 ${ }^{8-11}$. Cellular functions of Msd1 and Wdr8 in plant cells, which lack centrosomes, are not known.

In plant interphase cells, nascent microtubules are predominantly nucleated from lattice-bound $\gamma$ TuRC complexes on preexisting cortical microtubules, either as a dominant branchforming pattern with a branch angle of $\sim 40^{\circ}$ or as a less frequent bundle-forming parallel nucleation manner ${ }^{12-14}$. The katanin complex, composed of the p60 AAA ATPase catalytic subunit and the $\mathrm{p} 80$ regulatory subunit ${ }^{2}$, is required to sever the minus end of daughter microtubules at the branch junction sites and release them from the mother microtubules to generate free microtubules that migrate on the cell cortex by polymer treadmilling ${ }^{14,15}$. Katanin also severs microtubules at intersections where they cross over each other on the plant cell cortex ${ }^{16,17}$, a process that promotes amplification and re-ordering of the cortical arrays in response to blue light stimulus ${ }^{18}$. Although the $\mathrm{p} 80$ regulatory subunit of katanin is required to target the p60 catalytic subunit to subcellular locations ${ }^{19}$, it is not known whether specific factors differentially recruit katanin to these distinct subcellular sites in plant cells. During plant cell division, branched microtubules are nucleated along the lattice of preexisting microtubules in the plant cytokinetic phragmoplast array ${ }^{20}$. Katanin mutations affect organization and positioning of the spindle and the phragmoplast ${ }^{21,22}$ but severing events are yet to be analyzed due to the high densities of mitotic microtubules. In this study, we show that the plant Msd1-Wdr8 complex is used not only to stabilize nucleation sites on cortical microtubule arrays but also to subsequently recruit katanin to these subcellular locations to sever daughter microtubules.

\section{Results}

The Msd1-Wdr8 complex is localized at cortical nucleation sites in Arabidopsis cells. We first examined whether Msd1 and Wdr8 of Arabidopsis thaliana form heteromeric complexes as reported in non-plant organisms. In Arabidopsis, two Msd1 orthologs ( $M s d 1 a$ and $M s d 1 b$ ) encode proteins with $75 \%$ identity and $86 \%$ similarity in amino acid sequences, and are expressed in highly overlapping cell types (http://travadb.org/), indicating high functional redundancy. A yeast two-hybrid assay was used to detect direct interactions between Msd1a (At5g57410), Msd1b (At2g18876), and Wdr8 (At5g07590). The results showed that Msd1a and Msd1b interacted with Wdr8 (Supplementary Fig. 1a). Strong auto-activation of $\mathrm{Wdr} 8$ as a bait precluded interaction assays between $\mathrm{Wdr} 8$ and its potential association partners. Next, we immunoprecipitated interacting proteins using a green fluorescent protein (GFP) antibody from transgenic Arabidopsis plants stably expressing GFP, Msd1b-GFP, or Wdr8-GFP (Fig. 1a and Supplementary Fig. 1b). From the Msd1b-GFP-expressing plants, Msdla, Msdlb, and Wdr8 were identified in the immunoprecipitates by liquid chromatography-tandem mass spectrometry (LC-MS/MS), in addition to Msd1b-GFP. Similarly, immune-precipitates from plants expressing Wdr8-GFP were also enriched in Msd1a, Msd1b, and Wdr8. These studies suggest that the in vivo protein complexes contain multiple copies of Wdr8 and Msd1, which may consist of either Msd1a, Msd1b, or both isoforms.

Previously, when GFP-fusions of putative Arabidopsis microtubule-associated proteins were transiently overexpressed in onion epidermal cells, Msd1a-GFP was localized along the lattice of cortical microtubules, whereas Wdr8-GFP was mostly found in the cytosol ${ }^{23}$. In this study, we expressed GFP-fusions of Msdla, Msd1b, and Wdr8 under the regulation of their native promoters in transgenic Arabidopsis plants that expressed mCherry- $\beta$-tubulin 6 (TUB6). In pavement cells of cotyledons, GFP-fusions of Msd1a, Msd1b, and Wdr8 were all localized as relatively stable punctate particles on cortical microtubules (Fig. 1b). However, averaged projections of 151 frames (302 s) revealed that both Msd1 fusions also labeled the microtubule lattice, while a diffuse cytosolic pattern was revealed for Wdr8GFP. These similar but distinct subcellular localizations of Msd1 and Wdr8 reflect our previous observations in the transient overexpression studies ${ }^{23}$ and are consistent with the identification of Xenopus laevis SSX2IP (an ortholog of Msd1) as a microtubuleassociated protein ${ }^{5}$.

To gain further insights into the microtubule-related processes in which the plant Msd1-Wdr8 complex is involved, we investigated the localization of Msd1-Wdr8 in relation to the dynamics and nucleation events of cortical microtubules, as visualized with co-expression of mCherry-TUB6 and GFP-fusions of Msd1a, Msd1b, or Wdr8 (Fig. 1c, d and Supplementary Movies 1-4). In Arabidopsis interphase cells, microtubules are predominantly nucleated from $\gamma$ TuRCs that are recruited along the preexisting cortical microtubules ${ }^{12,14}$. Large fractions of Msd1-GFP and Wdr8-GFP puncta were observed at cortical nucleation sites (Msd1a, 47.4\%, $n=962$; Msd1b-GFP, 59.1\%, $n=$ 1198; and Wdr8-GFP, $60.7 \%, n=1149)$. Most nucleation siteassociated puncta were observed at branching nucleation sites (73-83\%), followed by bundle-forming sites (parallel with mother microtubules, 15-23\%) and a small number at free nucleation sites (<4\%) (Fig. 1e). This distribution of localization is similar to the proportion of each type of nucleation site in these arrays ${ }^{14,24}$ and highly similar to that of MOZART1a-GFP ${ }^{25}$ (MZT1-GFP), an integral component of the $\gamma$ TuRC and essential for microtubule nucleation in Arabidopsis ${ }^{25}$. Punctate localization of Msdla-GFP at nucleation sites was abolished in $w d r 8$ cells $(69$ events observed from 3 cells) and likewise for Wdr8 in msd1a msd1b double mutant cells (54 events observed from 3 cells) (Fig. 1c, d, Supplementary Figs. 2 and 3, and Supplementary Movies 5-8). Together, these observations show that Msd1 and Wdr8 localize to cortical microtubule nucleation sites, and that the function of both Msd1 and Wdr8 is required for such localization, supporting the idea that the observed punctate signals represent the heterodimeric complexes of Msd1 and Wdr8, and that stable localization to cortical nucleation sites requires complex formation.

To precisely determine the temporal localization of the Msd1-Wdr8 complex with respect to $\gamma$ TuRC, we co-expressed Msdla-mCherry and MZT1-GFP in transgenic Arabidopsis plants. Confocal imaging of epidermal cells in the hypocotyl of dark-grown seedlings revealed that predominant portions of the Msdla labels (99.0\% in 110 particles in 3 cells) and the MZT1 labels $(90.1 \%$ in 121 particles in 3 cells) colocalized with the other labels (Fig. 1f). When the exact timing of their cortex appearance was examined, the majority of Msdla particles $(76.8 \%$ in 341 
a

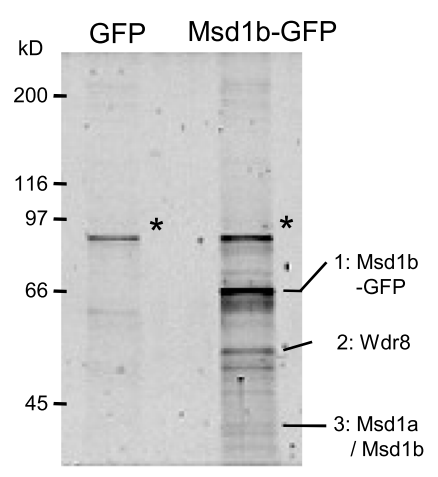

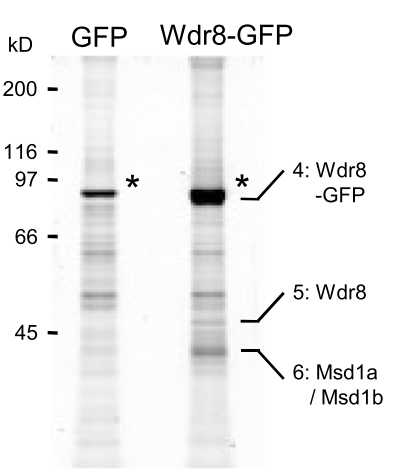
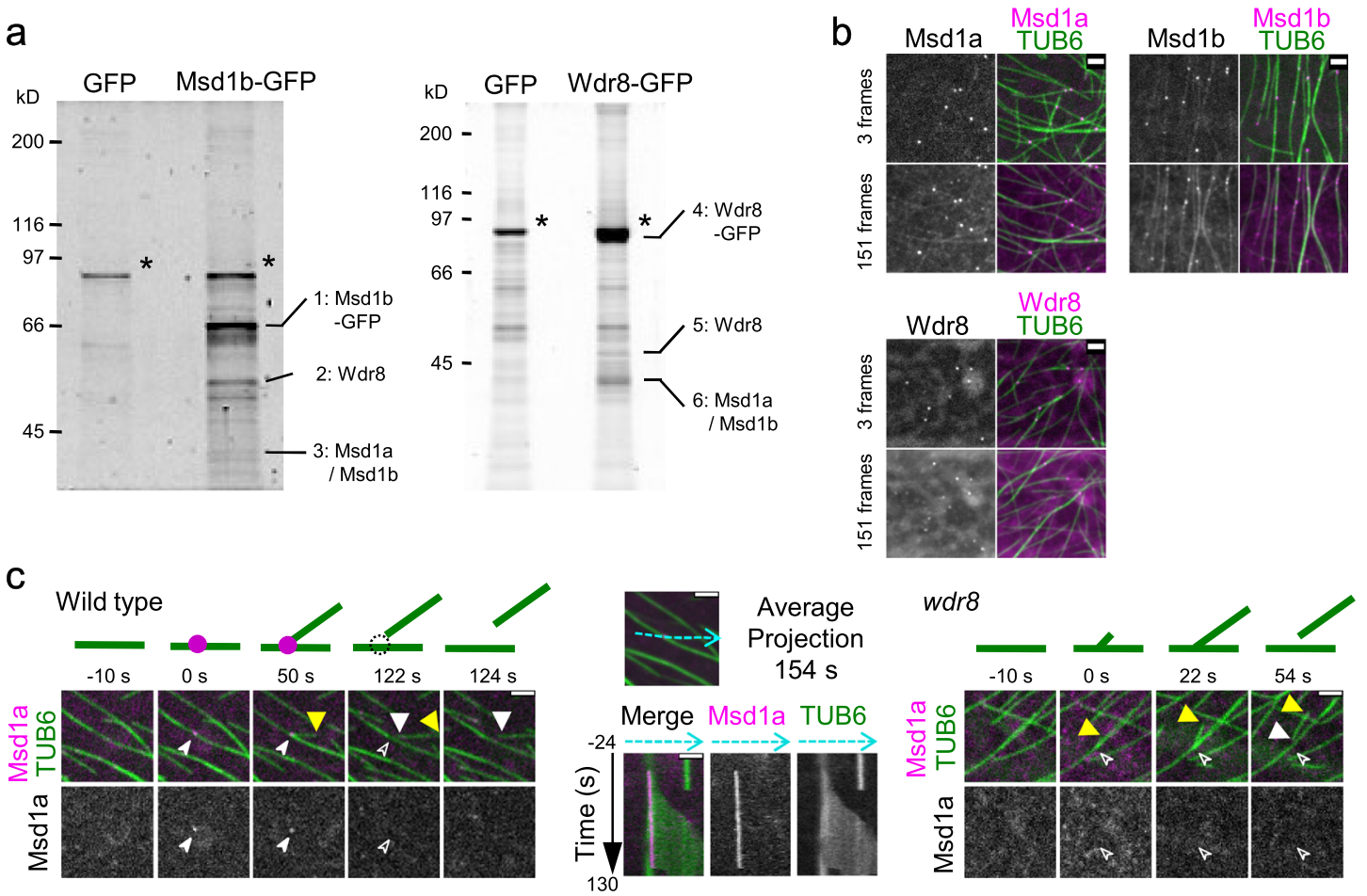

d
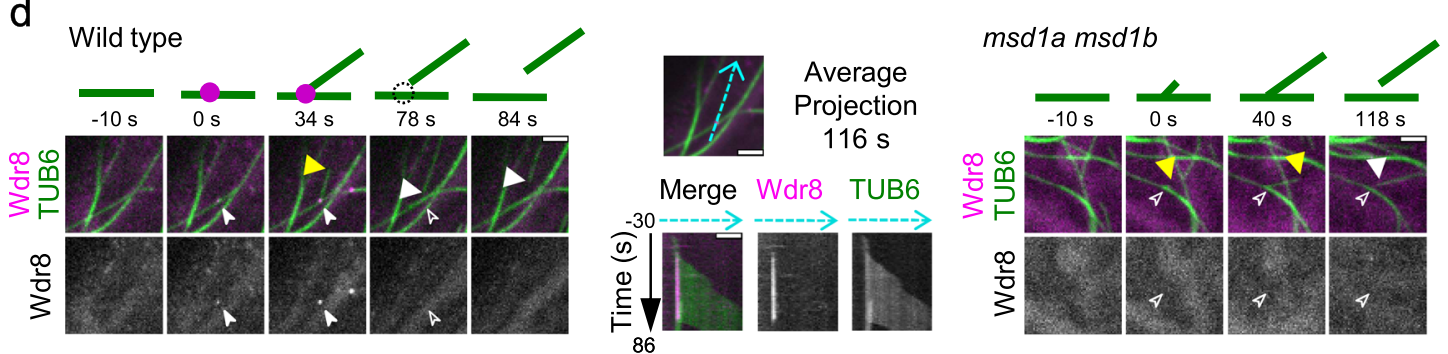

e

\begin{tabular}{|c|c|c|c|c|c|c|}
\cline { 2 - 7 } \multicolumn{1}{c|}{} & \multicolumn{2}{|c|}{ No Nucleation } & \multicolumn{3}{c|}{ Nucleation } & \multicolumn{1}{c|}{} \\
\cline { 2 - 7 } \multicolumn{1}{c|}{} & $\begin{array}{c}\text { O } \\
\text { Along } \\
\text { MT }\end{array}$ & $\mathbf{O}$ & $\mathbf{O}_{\mathbf{N}}$ & $\mathbf{O}=$ & $\mathbf{Q}$ & $\begin{array}{c}\text { No. of } \\
\text { particles } \\
\text { analyzed }\end{array}$ \\
\hline Msd1a & $52.1 \%$ & $0.5 \%$ & $37.7 \%$ & $7.8 \%$ & $1.9 \%$ & 962 \\
\hline Msd1b & $40.7 \%$ & $0.3 \%$ & $48.8 \%$ & $8.7 \%$ & $1.6 \%$ & 1198 \\
\hline Wdr8 & $37.9 \%$ & $1.3 \%$ & $44.0 \%$ & $14.0 \%$ & $2.7 \%$ & 1149 \\
\hline MZT1 & $\mathbf{3 6 . 3} \%$ & $\mathbf{0 . 0} \%$ & $\mathbf{5 0 . 8} \%$ & $\mathbf{1 2 . 8} \%$ & $\mathbf{0 . 1} \%$ & ref. $\mathbf{2 5}$ \\
\hline
\end{tabular}
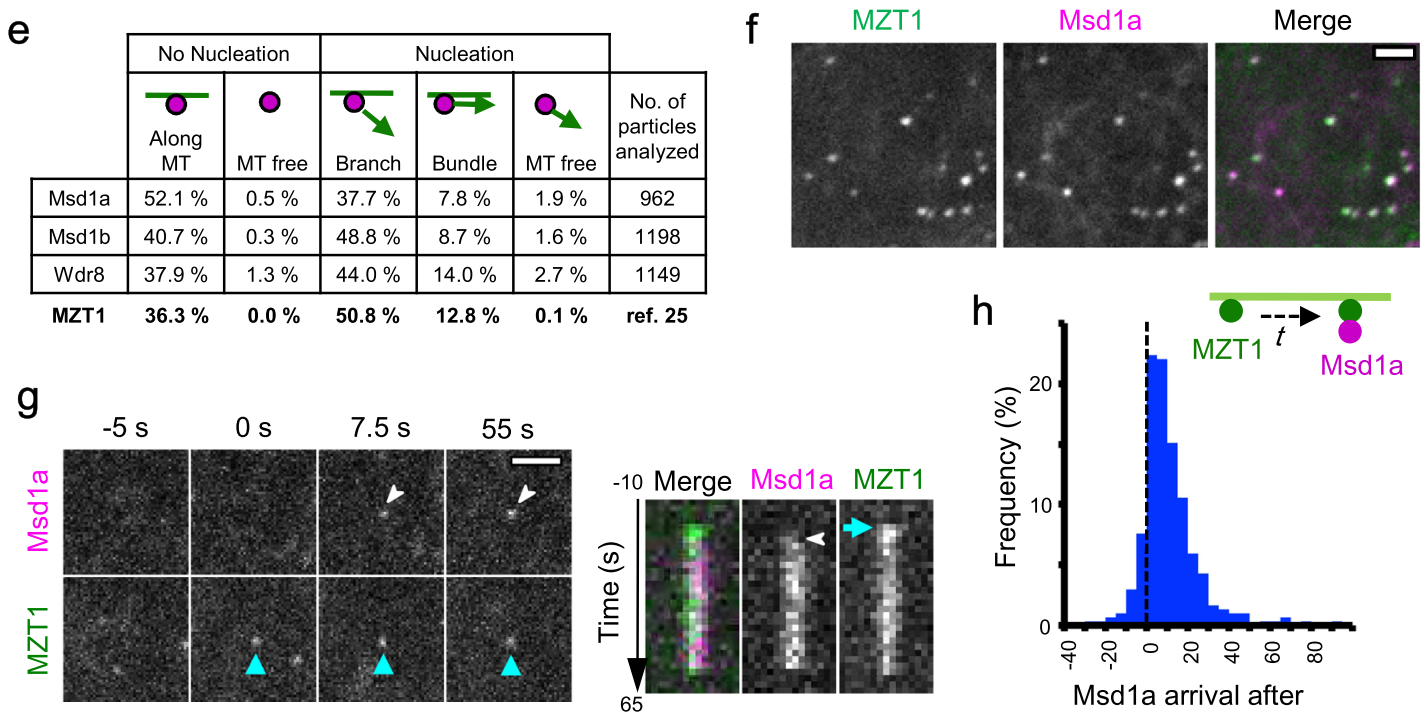

particles in 6 cells) appeared after a short delay to cortical locations where MZT1-labeled $\gamma$ TuRCs had already been recruited (mean time lag of $13.7 \pm 2.8 \mathrm{~s}$, Fig. $1 \mathrm{~g}$, $\mathrm{h}$ and Supplementary Movie 9). Both labels disappeared from the cell cortex approximately at the same time. These results suggest that shortly after $\gamma$ TuRC is recruited to cortical microtubules, the
Msd1-Wdr8 complex becomes stably associated with it. The Msd1 ortholog in fission yeast is reported to interact with Alp4, a component of $\gamma \mathrm{TuRC}^{4}$.

Release of daughter microtubules is delayed in $m s d 1$ and $w d r 8$ mutant cells. We found that the mean life time of MZT1-GFP 


\begin{abstract}
Fig. 1 The Msd1-Wdr8 complex associates with the $\boldsymbol{\gamma}$-tubulin ring complex at cortical nucleation sites along the microtubule lattice. a Immuno pulldown experiments of Msd1- or Wdr8-interacting proteins from Arabidopsis seedlings. Seedlings stably expressing either GFP, Msd1b-GFP, or Wdr8-GFP were used to prepare cell extracts for immunoprecipitation using GFP-antibody beads. Precipitated proteins were separated by SDS-PAGE and detected by staining with Flamingo fluorescent dye. Asterisks indicate major nonspecific bands. This experiment was repeated twice with similar results. b Subcellular localizations of Msd1a-GFP, Msd1b-GFP, and Wdr8-GFP in cotyledon pavement cells when expressed under their native promoters. The TUB6 marker labels microtubules. Snap shots ( 3 frames) and integrated exposures of 151 frames ( $302 \mathrm{~s}$ total time) are shown. c, d Recruitment of Msd1a-GFP particles (c) and Wdr8-GFP particles (d) to the branch-forming nucleation sites on cortical microtubules in wild-type (left), wdr8 (c, right), and msdla msd7b (d, right) cells. Time-lapse confocal microscopy images are shown at the indicated times. For wild-type cells, kymographs of Msdla-GFP (c) or Wdr8-GFP (d) and microtubules were generated along the dotted blue lines in the average projection images of $154 \mathrm{~s}$ and $116 \mathrm{~s}$, respectively. Open and closed arrowheads indicate the absence and the presence, respectively, of Msdla or Wdr8 particles. Likewise, the yellow and white triangles show the plus and minus ends of daughter microtubules, respectively. The events occurring at the indicated time points are schematically presented with microtubules (green lines) and Msd1-Wdr8 particles (magenta circles). e Localization of Msd1a-GFP, Msd1b-GFP, and Wdr8-GFP in relationship to microtubule nucleation was classified into five event groups. Percentages of events observed in five groups and the total number of observed events are shown. $\mathbf{f}$ Colocalization of Msd1a$\mathrm{mCherry}$ and MZT1-GFP particles on the cell cortex region in Arabidopsis hypocotyl cells in the average projection images of $62.5 \mathrm{~s}$. More than 110 particles from three cells were observed with similar results. $\mathbf{g}$ Recruitment of Msd1a-mCherry and MZT1-GFP particles. Left: time-lapse confocal microscopy images at the indicated times. Right: kymograph generated from the time-lapse microscopy images shown in the left. $\mathbf{h}$ Distribution of the arrival times ( $t$ in the diagram) of Msd1a-mCherry particles (magenta) after the appearance of MZT1-GFP particles (green) on the cell cortex ( $n=304$ events from 6 cells). The MZT1 particles arrive prior to the Msd1 particles. Bars, $2 \mu \mathrm{m}$.
\end{abstract}

particles at the cell cortex is considerably longer in the msd1a $m s d 1 b$ cells and the $w d r 8$ cells than in control cells (Fig. 2a and Supplementary Movie 10). The MZT1-GFP particles on the cortical microtubules that did not result in microtubule nucleation disappeared in a relatively short time (Fig. 2b); the residence time of these non-nucleating complexes did not significantly differ among wild-type cells $(14.0 \pm 20.7 \mathrm{~s}, n=652$ particles), msd1a msd1b cells (7.9 $\pm 13.7 \mathrm{~s}, n=554$ particles), and $w d r 8$ cells $(12.1 \pm 21.3 \mathrm{~s}, n=340$ particles $)$. By contrast, the microtubule-nucleating MZT1-GFP particles in the mutant cells remained on cortical microtubules longer than those in the wild-type cells (Fig. 2b). The proportions of the nucleation particles that persisted longer than $160 \mathrm{~s}$ were $3.4 \%$ in wild-type cells $(n=378), 39.4 \%$ in msdla msd1b cells $(n=226)$, and $30.7 \%$ in $w d r 8$ cells $(n=215)$. To dissect nucleation and release events, we measured the time to nucleate microtubules after MZT1-GFP particles appeared on the lattice of cortical microtubules (Fig. 2c). The nucleation time was not significantly different among wild-type cells $(20.6 \pm 16.9 \mathrm{~s}, n=235$ particles $)$, $m s d 1 a$ msd $1 b$ cells $(25.5 \pm 19.7 \mathrm{~s}, n=59$ particles $)$, and $w d r 8$ cells $(17.9 \pm 14.4 \mathrm{~s}, n=76$ particles). Thus, in the mutant cells, nucleated $\gamma$ TuRCs persisted much longer on mother microtubules than in wild-type cells.

Subsequent to nucleation of the daughter microtubule, the $\gamma$ TuRC in wild-type cells is destabilized and disappears from the mother microtubule by two distinct mechanisms ${ }^{14}$; release of the minus end of the daughter microtubule (Fig. 2d and Supplementary Movie 11) and complete shrinkage of the dynamic plus end of the daughter microtubule (Fig. 2e and Supplementary Movie 12). In wild-type cells, release events were observed to predominate (67.5\% in 449 particles) over shrinkage events. By contrast, the majority of MZT1-GFP disappearance was caused by microtubule shrinkage in $m s d 1 a$ msd1b cells $(74.9 \%$ in 223 particles) and $w d r 8$ cells ( $80.2 \%$ in 364 particles) (Fig. 2f). The observed shift of MZT1-GFP disappearance might be explained if microtubule release was less likely or more delayed in the mutant cells. To test this idea, we measured the time distribution of daughter microtubule release after nucleation. The release of daughter microtubules took a mean time of $63.8 \pm 32.3 \mathrm{~s}(n=235$ events) in wild-type cells, whereas the mean release time increased significantly to $130.8 \pm 80.3 \mathrm{~s}$ ( $n=59$ events) in $m s d 1 a$ $m s d 1 b$ cells and $157.7 \pm 171.3 \mathrm{~s}(n=76$ events $)$ in $w d r 8$ cells (Fig. 2g). Thus, extended persistence of $\gamma$ TuRC complexes observed in $m s d 1$ and $w d r 8$ cells likely results from the delayed release of daughter microtubules from cortical nucleation sites, implying that the Msd1-Wdr8 complex functions to promote daughter release.

Recruitment of katanin to cortical nucleation sites requires Wdr8. In interphase plant cells, katanin is known to sever microtubules at branching nucleation sites ${ }^{14,15}$. Therefore, a reasonable mechanism for Msd1-Wdr8 action on daughter microtubule release might be to recruit katanin or stimulate katanin activity. To investigate these possibilities, we first asked whether katanin is recruited to the subcellular sites where Msd1-Wdr8 particles have been localized. When dynamics of GFP-KTN1 (a GFP fusion of the p60 catalytic subunit, expressed from native upstream sequences) and Msdla-mCherry were simultaneously followed in the wild-type cells, some GFP-KTN1 particles and some Msdla-mCherry particles were transiently colocalized at the cell cortex (Supplementary Fig. 1 and Supplementary Movie 13). In these instances, the KTN1 particles arrived at the cortical locations where the Msdla particles had already been tethered with a mean lag time of $19.1 \pm 21.5 \mathrm{~s}$ ( $n=90$ events from 3 cells), indicating transient association of Msd1-Wdr8 with katanin.

Next, we examined whether katanin recruitment to branching nucleation sites is affected in the $w d r 8$ mutant cells. After nucleation of a daughter microtubule on the lattice of a mother microtubule in wild-type cells, fluorescence signals of GFP-KTN1 gradually increased at the base of the branched microtubules. Initial accumulation of GFP-KTN1 was weak and fluctuating, but over time the signal intensity incremented and peaked just before the observed detachment of the minus end (Fig. 3a, b, d and Supplementary Movie 14). The observation that GFP-KTN1 signal at nucleation sites was initially low and fluctuating before reaching a peak suggests that multiple copies of the katanin hexamer may be needed to completely sever microtubules from these sites. In many cases (67\% of 30 events from 3 cells), GFPKTN1 signals dissipated before detection of the minus-end shortening, or the two events were observed at the same timelapse frames (30\%). As the slowly depolymerizing minus end needs to shorten sufficiently to resolve optically the minus end from the mother microtubule, signal loss due to release of katanin complexes therefore likely occurred at or shortly after the minusend detachment. Some GFP-KTN1 puncta (60\% of 30 events from 3 cells) were dislodged from the nucleation sites prior to disappearance (Fig. 3b, arrowheads), indicating that association of katanin to the nucleation sites is destabilized shortly after the minus-end severing. By contrast, in the $w d r 8$ cells, GFP-KTN1 

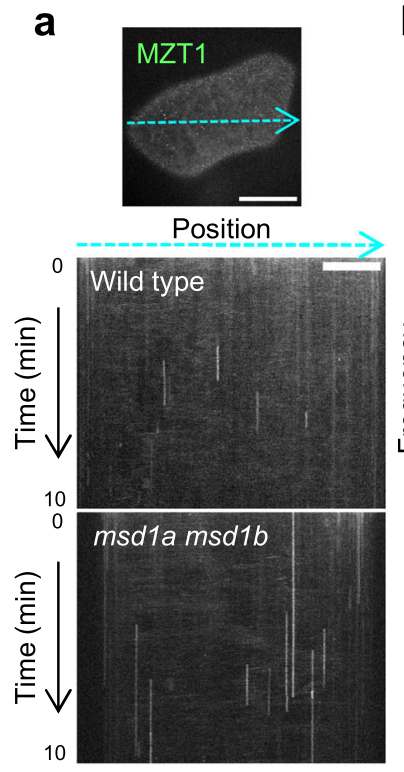

d Microtubule release
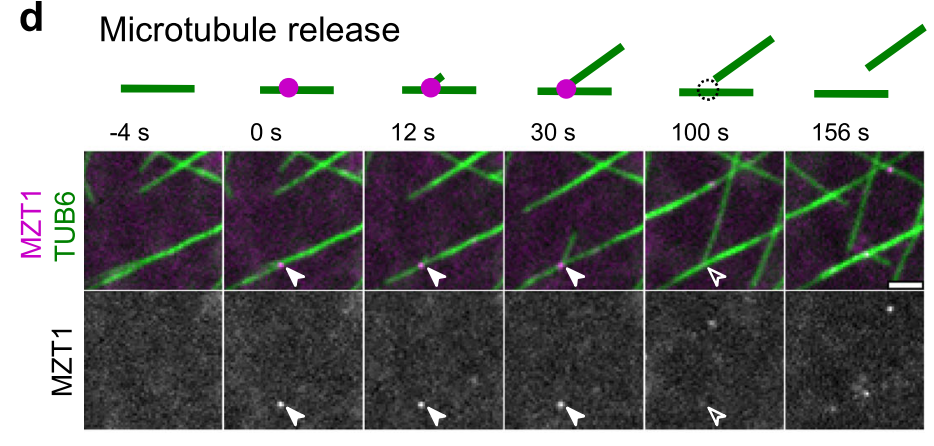

e

Microtubule shrinkage

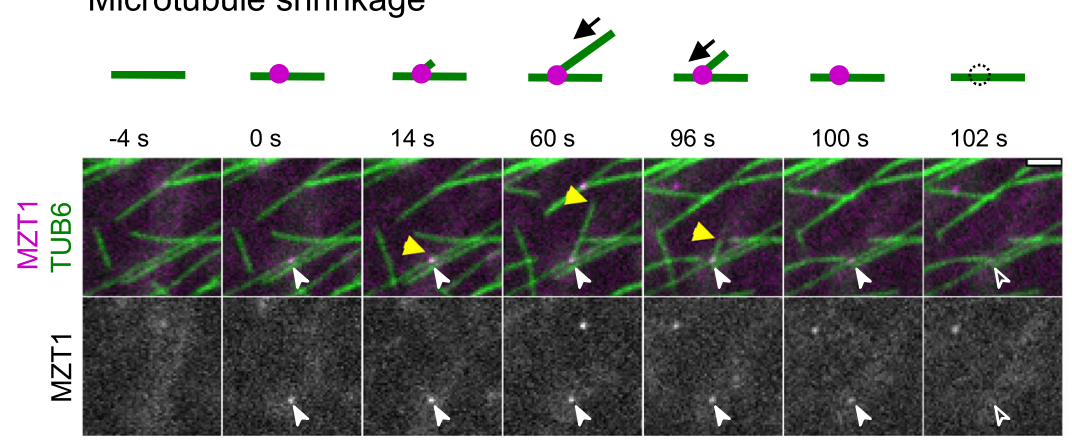

b No MT nucleation MT nucleation
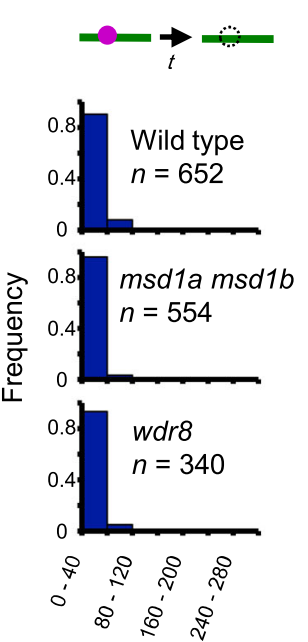

Residence time (s)

Residence time (s)
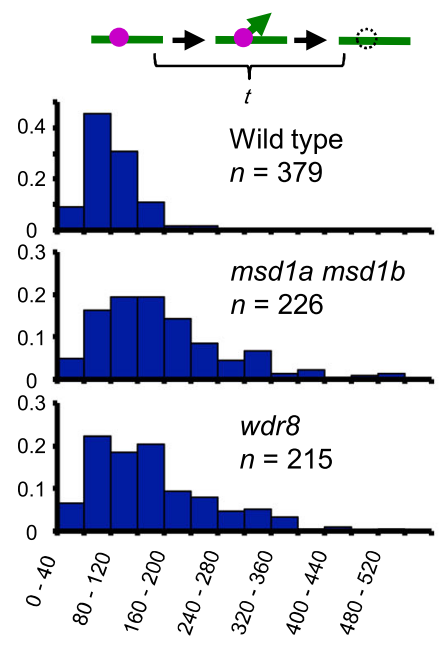

did not accumulate at the base of branched microtubules above the background level (Fig. 3c-e and Supplementary Movie 15). Thus, Wdr8 is required for katanin recruitment to the branching nucleation sites, explaining delayed daughter microtubule release.

As Msdla-GFP, Msd1b-GFP, and Wdr8-GFP are all recruited to the bundle-forming nucleation sites in wild-type cells (Fig. 1e; also see Supplementary Fig. 5 and Supplementary Movies 16-18), we asked whether Msd1-Wdr8 is also necessary for katanin recruitment to these nucleation sites. Although bundle-forming nucleation is technically difficult to identify in cells expressing both mCherry-TUB6 and GFP-KTN1, we managed to find several such events with confidence (seven events in wild-type cells and three events in $w d r 8$ cells). In wild-type cells, GFP-KTN1 arrived at the
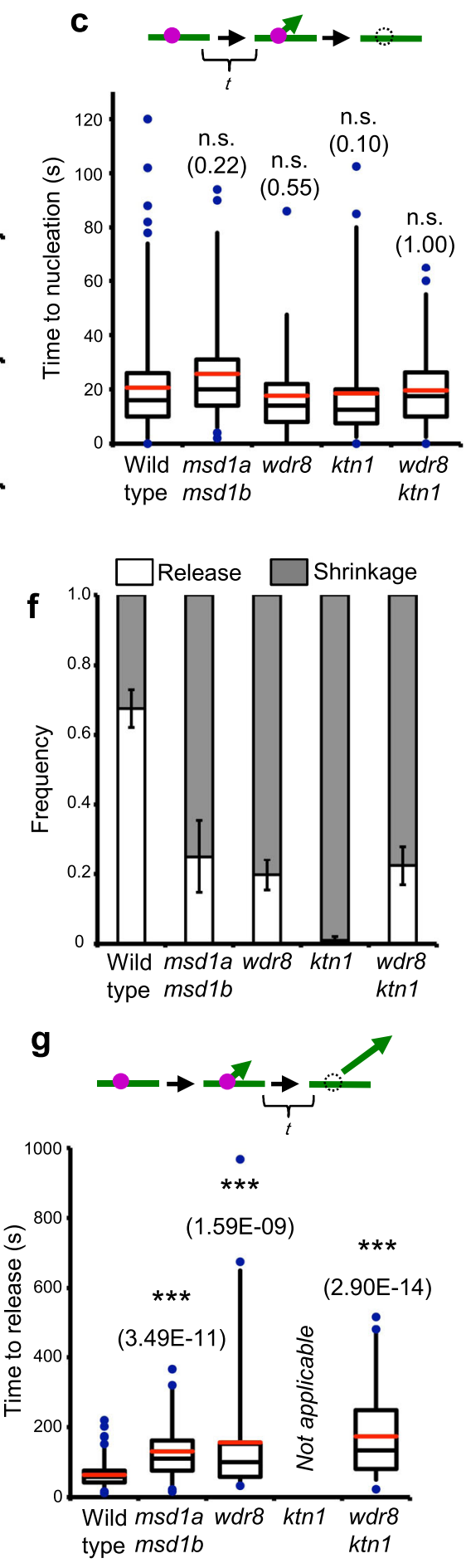

bundle-forming nucleation sites dozen seconds after nucleation in all seven cases (Fig. $3 \mathrm{f}$ and Supplementary Movie 19). In contrast, in $w d r 8$ cells, GFP-KTN1 particles were not detected at the minus end of daughter microtubules during the period of parallel microtubule nucleation and subsequent release (Fig. $3 \mathrm{~g}$ and Supplementary Movie 20). Therefore, daughter microtubules nucleated by either branching or bundle-forming manner require Wdr8 to recruit katanin.

The Msd1-Wdr8 complex stabilizes the association of daughter microtubule minus ends to their nucleation sites. Despite the lack of detectible GFP-KTN1 recruitment at nucleation sites in the $w d r 8$ 
Fig. 2 Mutations of the Msd1-Wdr8 complex delay the time to daughter microtubule release after nucleation. a Persistence of $\gamma$ TuRC, as labeled with MZT1-GFP, on the cell cortex region was followed over $10 \mathrm{~min}$ by time-lapse confocal microscopy in cotyledon pavement cells. Kymograph analysis was performed along images of the cell cortex as exemplified by the blue dotted line in this wild-type cell. Kymographs of a wild-type cell and an msd7a msd1b mutant cell are shown. Bar, $20 \mu \mathrm{m}$ in the panel image and $10 \mu \mathrm{m}$ in the kymograph. Three cells were analyzed with similar results. $\mathbf{b}$ Distribution of the residence times ( $t$ in the diagram) of the MZT1-GFP particles (magenta circles) on the mCherry-TUB6-labeled cortical microtubules (green lines) in wild type (seven cells), msdia msdlb (four cells), and wdr8 (five cells). The particles were classified into those that disappeared without or after microtubule nucleation. In the mutant cells, nucleating $\gamma$ TuRC particles tend to stay longer on the microtubule lattice. $\mathbf{c}$ Times to nucleate nascent microtubules ( $t$ in the diagram) from the lattice-bound $\gamma$ TuRC (magenta circles) in wild type ( 235 events from 6 cells), msd1a msd1b (58 events from 6 cells), and wdr8 (76 events from 9 cells), $k \operatorname{tn} 1$ (177 events from 4 cells), and wdr $8 \mathrm{ktn} 1$ ( 66 events from 4 cells). Nucleating times of the mutants are not significantly different from that of wild type ( $p>0.09$, determined by the Steel-Dwass test). The bottom and top of each box represent the first and third quartiles, respectively, the horizontal line inside each box is the median (second quartile) and the red horizontal line represents the average. The whiskers range between 2 nd percentile and 98 th percentile. Data points outside this range are considered as outliers and are indicated as circles. n.S., not significant. Exact $p$-values are indicated in parentheses. d, e Time-lapse confocal microscopy images of the minus-end release event (d) and the complete shrinkage event from the plus end (e) of daughter microtubule at the indicated times in wild-type cells. $\gamma$ TuRC (magenta circles in the diagrams) and microtubules (green lines) are labeled by MZT1-GFP and mCherry-TUB6, respectively. Open and closed arrowheads and yellow triangles respectively indicate the absence and the presence of MZT1 particles and the plus ends of daughter microtubules. Bars, $2 \mu \mathrm{m}$. $\mathbf{f}$ Proportions of the MZT1 particle disappearance caused by the minusend release (green) and the plus-end shrinkage (magenta) in wild-type (449 events from 7 cells), msd7a msd1b (223 events from 6 cells), and wdr8 (367 events from 7 cells), ktn1 (301 events from 4 cells), and wdr8 ktn1 (294 events from 4 cells). The error bars indicate SD. $\mathbf{g}$ Times ( $t$ in the diagram) for daughter microtubules to be released from mother microtubules after nucleation. The event numbers for each genotype are the same with $\mathbf{c}$. In the diagram, a daughter microtubule grows at the plus end (arrowhead) after nucleation from $\gamma$ TuRC (magenta). ${ }^{\star \star \star} p<0.0001$, determined by the Steel-Dwass test. Exact $p$-values are indicated in parentheses. The analysis is not applicable for the ktn 1 cells where the release events are very rare. The elements of the box plot are defined as in $\mathbf{c}$.

mutant cells, the minus ends of daughter microtubules were eventually detached, indicating that katanin is not absolutely required for the minus-end detachment in the $w d r 8$ cells. This observation was surprising, as our previous investigations ${ }^{14,15}$ had shown that katanin was required for the release of daughter microtubules from nucleation complexes by severing. To further characterize the katanin-independent microtubule detachment events observed in the $w d r 8$ cells, we directly compared microtubule release events in $\mathrm{p} 60$ katanin mutant $(k t n 1)$ cells and $w d r 8$ $k t n 1$ double mutant cells. Consistent with previous observations, in the $k t n 1$ cells, daughter microtubules generally remained attached to the lattice of mother microtubules at the branched nucleation sites during the observation period of $>15 \mathrm{~min}$, unless the mother microtubule depolymerized (Fig. 4a and Supplementary Movie 21). In rare cases, daughter microtubules were found to be released eventually (three detachments events observed after 125, 163, and $750 \mathrm{~s}$ of microtubule nucleation in a total of 301 branching nucleation events observed), concomitant with a disappearance of the MZT1-GFP nucleation complex marker (Supplementary Fig. 6 and Supplementary Movie 22). In the $w d r 8 \mathrm{ktn} 1$ cells, on the other hand, daughter microtubules were now readily released (Fig. 4b and Supplementary Movie 23), along with loss of MZT1-GFP (22.4\% of observed branching nucleation events over the observation interval). This rate of daughter detachment and MZT1-GFP loss in the absence of daughter microtubule shrinking was comparable to that observed in the $w d r 8$ single mutant cells $(19.8 \%)$ (Fig. 2f). The MZT1-GFP particles were released in conjunction with daughter detachment after $174.2 \mathrm{~s}( \pm 123.7 \mathrm{~s}$ in 63 events $)$ of microtubule nucleation in the $w d r 8 \mathrm{ktn} 1$ cells, which was also comparable with the release time in the $w d r 8$ cells (Fig. $2 \mathrm{~g}$ ). These results indicate that $\mathrm{Wdr} 8$ is required to stabilize the nucleated branching sites on cortical microtubules. Without Wdr8, the branched nucleation sites readily disintegrate and the minus ends of daughter microtubules are released even in the absence of the severing activity of katanin. Thus, it appears that $\mathrm{Wdr} 8$ has two functions; it is needed to stabilize the nucleated $\gamma \mathrm{TuRC}$ on the side of the mother microtubule and it is required to recruit katanin to detach the daughter microtubule after it is nucleated.

We further examined whether other nucleation characteristics of cortical microtubules are affected in the mutant cells. Nucleation frequencies per unit cortical area per unit time (sum of branching, bundle-forming, and free nucleation types) were not significantly different $(P>0.6$, Steel-Dwass test) between genotypes $(0.43 \pm 0.09$ per $100 \mu \mathrm{m}^{2}$ per min, $n=541$ from 7 wild-type cells, $0.47 \pm 0.22$ per $100 \mu \mathrm{m}^{2}$ per min, $n=355$ from $6 m d s 1 a m s d 1 b$ cells, and $0.42 \pm$ 0.24 per $100 \mu \mathrm{m}^{2}$ per min, $n=309$ from $5 w d r 8$ cells). In the branch-forming nucleation, the angles formed between mother microtubules and daughter microtubules were not significantly different between wild-type cells $(42.2 \pm 9.7, n=126$ events from 4 cells), msd $1 a$ msd $1 b$ cells ( $38.5 \pm 13.0, n=121$ from 5 cells), and $w d r 8$ cells ( $39.0 \pm 14.9, n=97$ from 5 cells) (Supplementary Fig. 7a). In the wild-type cells, the branch-forming nucleation predominates over the bundle-forming parallel nucleation ${ }^{13,14}$. In the msd1a $m s d 1 b$ cells $(n=355$ events from 6 cells) and $w d r 8$ cells $(n=309$ events from 5 cells), the relative proportion of the bundle nucleation type moderately but significantly increased over the branching nucleation type, compared to the wild-type cells $(n=541$ events from 7 cells) $(P<0.001$, Fisher's exact test) (Supplementary Fig. 7b, c). The Msd1-Wdr8 complex may have larger roles in stabilizing the branch-forming orientation, compared to parallel orientation, of $\gamma$ TuRC on the cortical microtubules.

Katanin recruitment during cell division does not require the Msd1-Wdr8 complex. Plant microtubules in mitotic arrays, spindles, and phragmoplasts, are also nucleated along preexisting microtubules in a $\gamma$ TuRC- and augmin-dependent manner ${ }^{26,27}$, and katanin is required for the organization of mitotic microtubule arrays and for proper progression of mitosis and cytokinesis $21,22,28$. Subcellular localization of Msd1a-mCherry and GFP-KTN1 during mitosis revealed partially overlapping but distinct distributions. In particular, Msdla-mCherry localized broadly on prophase and metaphase microtubules, whereas katanin mostly accumulated after anaphase and distributed more toward the spindle poles where the minus ends are enriched (Fig. 5a and Supplementary Movie 24). Katanin localization on mitotic microtubules was indistinguishable between wild-type cells and the $w d r 8$ mutant cells (Fig. 5b and Supplementary Movies 25 and 26). Therefore, Msd1-Wdr8 does not appear to play a major role for katanin recruitment on mitotic microtubules. A phragmoplast-localized plant-specific protein CORD4 contributes to tethering of katanin to the distal end of phragmoplast microtubules ${ }^{28}$. 
a Wild type

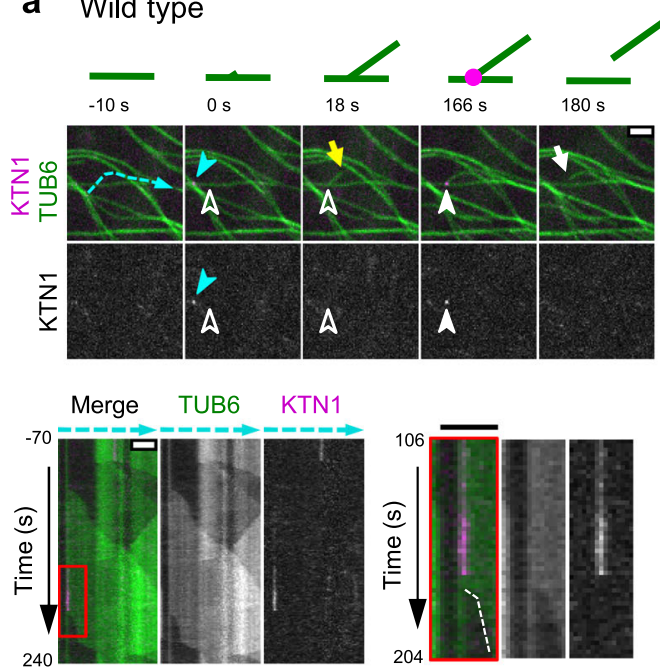

b KTN1 / TUB6 (wild type)

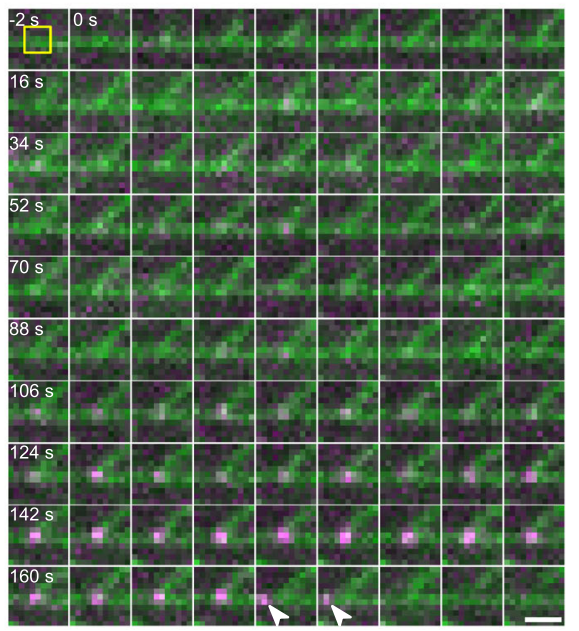

C $w d r 8$
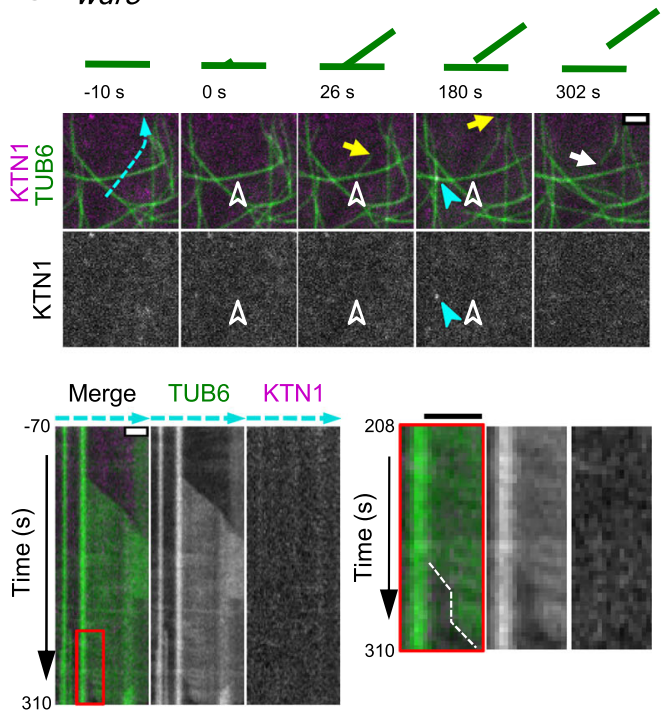

d
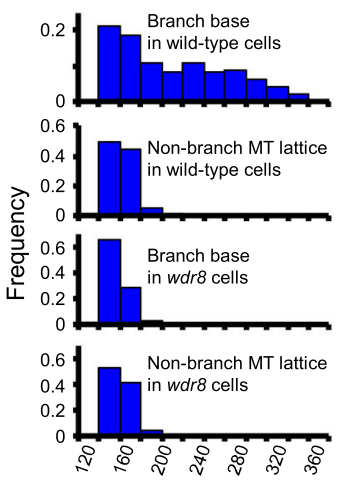

Max KTN1 intensity at ROI e

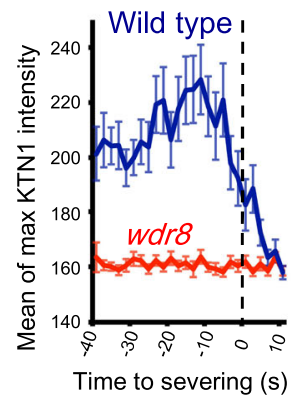

f Wild type
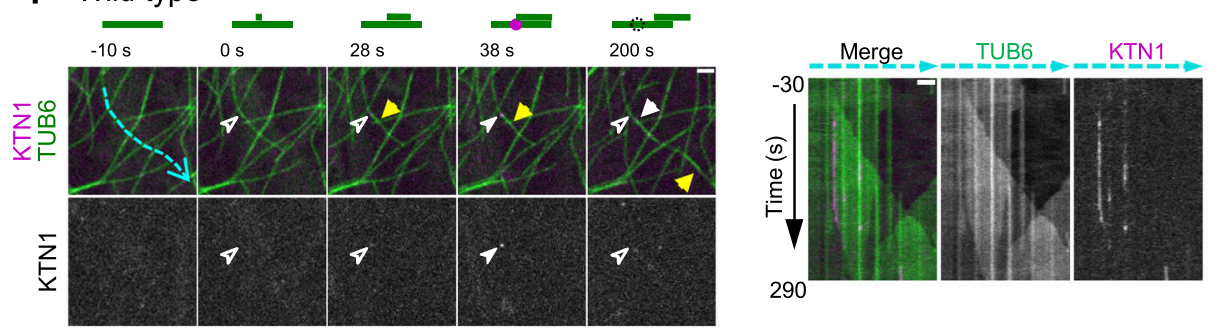

g $w d r 8$
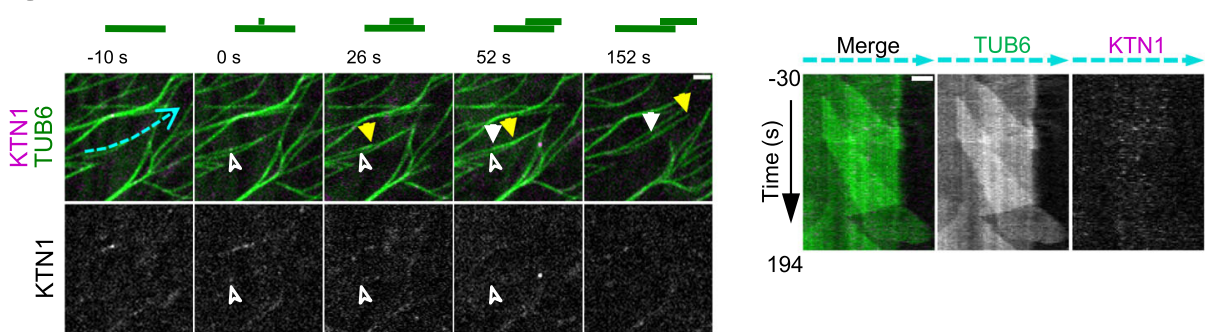

Katanin recruitment to microtubule crossover sites does not require the Msd1-Wdr8 complex. GFP-KTN1 was recruited also to microtubule crossover sites at the cell cortex (Fig. 6a-d and Supplementary Movies 27 and 28). Katanin consistently accumulated at crossover sites prior to severing, consistent with previous observations ${ }^{17,18}$. After severing, the new plus end first underwent rapid depolymerization. Slow depolymerization of the minus end was observed several seconds later $(5.4 \pm 5.6 \mathrm{~s}$ in 25 events), whereas GFP-KTN1 signal sometimes remained for several seconds at the severed crossover site. Recruitment of GFP- 
Fig. $3 \mathbf{W d r 8}$ is required to recruit katanin to the cortical nucleation sites. a, c Time-lapse confocal microscopy images of katanin recruitment to the branching nucleation sites at the release of daughter microtubules in wild-type (a) and $w d r 8$ (c) cells. Katanin (magenta circles in the diagrams) and microtubules (green lines) are labeled by GFP-KTN1 and mCherry-TUB6, respectively. Open and closed white arrowheads respectively indicate the absence and the presence of KTN1 particles, whereas yellow and white arrows show the plus and minus ends of daughter microtubules. Closed blue arrowheads point to KTN1 particles at the microtubule crossover sites. Kymographs track the dotted blue lines. Microtubule nucleation events set the zero time points. Rectangle regions boxed by red lines on the left kymographs are enlarged on the right where dotted white lines mark the minus ends of released daughter microtubules. Bars, $2 \mu \mathrm{m}$. The images in a are representative of 90 similar events, whereas those in c represent 29 similar events. b Time-lapse images of katanin (magenta) and microtubules (green) at $2 \mathrm{~s}$ intervals in wild-type cells. Microtubule nucleation occurs at $0 \mathrm{~s}$, whereas detachment of the daughter microtubule minus end is recognizable at $172 \mathrm{~s}$. White arrowheads indicate the katanin particles dislodged from the branch base. A yellow square at $-2 \mathrm{~s}$ shows ROI ( 5 pixel $\times 5$ pixel). Bar, $1 \mu \mathrm{m}$. The images are representative of 30 similar events. d Distribution of GFP-KTN1 signal intensities at the branching nucleation sites (384 ROls from 6 wild-type cells and 464 ROIs from 4 wdr8 cells) and the non-nucleating regions of the mother microtubule lattice ( 272 ROIs from 6 wild-type cells and 320 ROls from 4 wdr 8 cells). MT, microtubule. e Katanin accumulates at the branching nucleation sites prior to severing in wild-type cells (green line; 24 events from 6 cells) but not in wdr8 cells (magenta line; 29 events from 4 cells).

Detachment of the minus ends of daughter microtubules from the mother microtubules were visually detected from the confocal images and was set to the time zero. The error bars indicate SD. $\mathbf{f}, \mathbf{g}$ Time-lapse confocal microscopy images of katanin recruitment to the bundle-forming nucleation sites at the release of daughter microtubules in wild-type $(\mathbf{f})$ and $w d r 8(\mathbf{g})$ cells. Similar events were observed in nine wild-type cells (f) and three wdr8 cells (g). Kymographs on the right track the dotted blue lines. Symbols are the same as in $\mathbf{a}$ and $\mathbf{c}$. Bars, $2 \mu \mathrm{m}$.
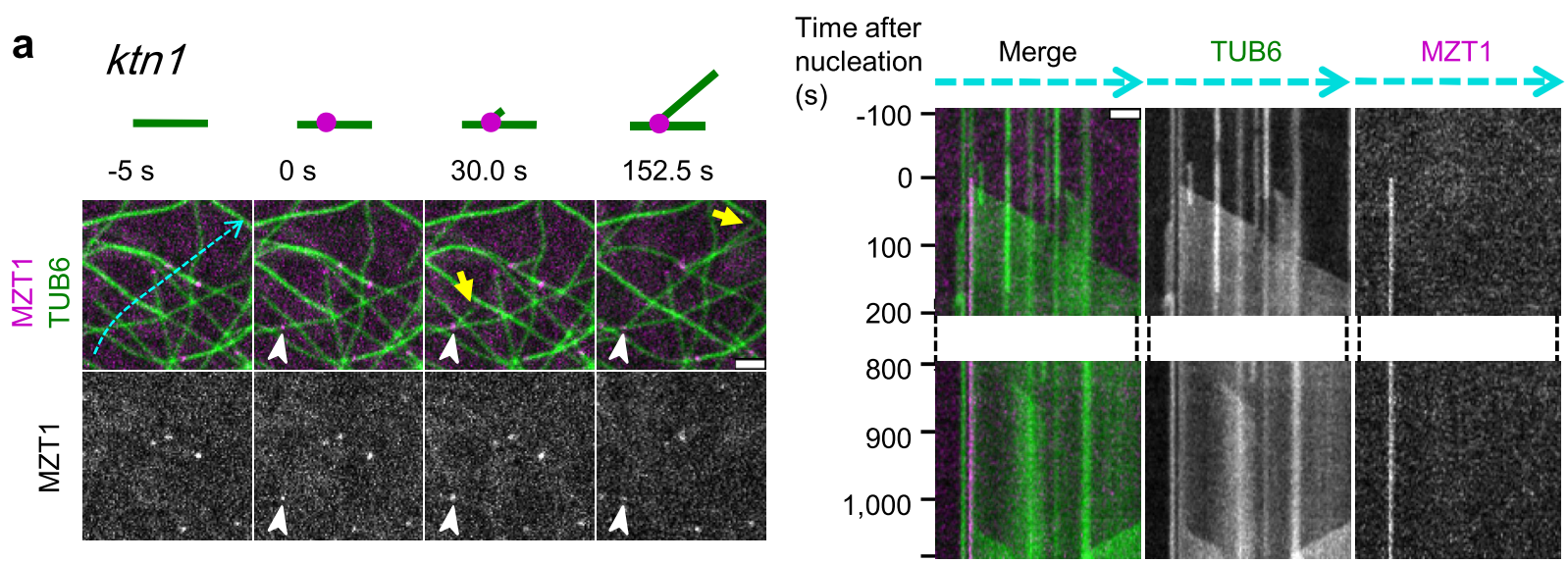

\section{b $\quad w d r 8 k \operatorname{tn} 1$}

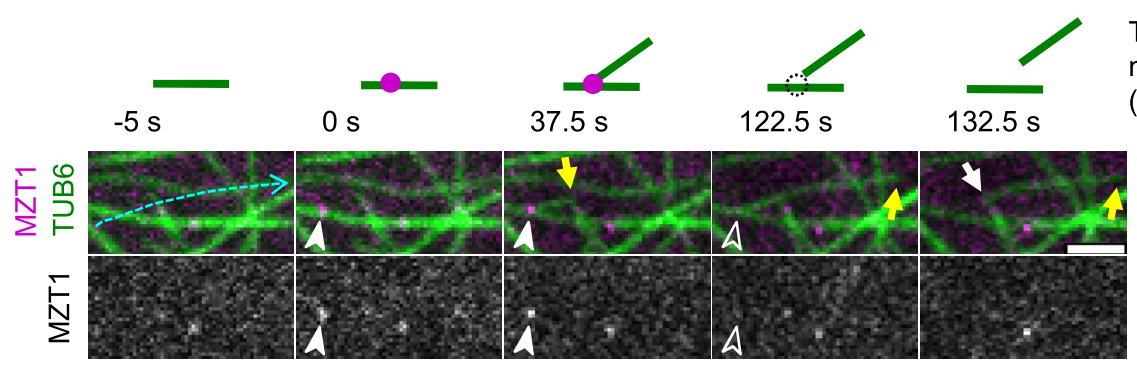

Time after nucleation

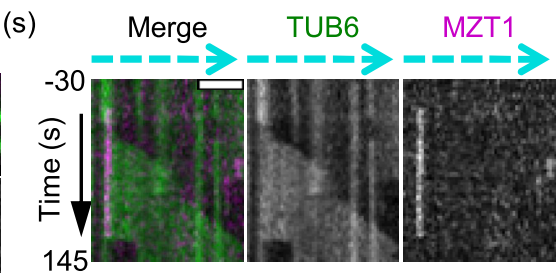

Fig. 4 Wdr8 stabilizes the branching nucleation sites. Time-lapse confocal microscopy images of the minus-end stability of daughter microtubules after branch-forming nucleation. $\gamma$ TuRC (magenta circles in the diagrams) and microtubules (green lines) are labeled by MZT1-GFP and mCherry-TUB6, respectively. Open and closed arrowheads respectively indicate the absence and the presence of MZT1 particles, whereas yellow and white arrows show the plus and minus ends of daughter microtubules. Kymographs track the dotted blue lines. Appearance of MZT1 particles set the zero time point. a In the $k t n 1$ cells, the minus ends of daughter microtubules are stably attached to the branching nucleation sites, as seen in the kymograph at $1100 \mathrm{~s}$ after nucleation. A total of 298 similar events were observed. $\mathbf{b}$ In the $w d r 8$ ktn 1 cells, daughter microtubules are readily released even in the absence of katanin. A total of 63 similar events were observed. Bars, $2 \mu \mathrm{m}$.

KTN1 and severing of crossed over microtubules did not differ between wild-type cells and the $w d r 8$ cells; $94.4 \%$ of the severed crossover sites in wild-type cells $(n=54,22$ cells) and $95.4 \%$ of the sites in the $w d r 8$ cells ( $n=65,20$ cells) showed evidence of prior accumulation of katanin. In support of this, microtubule crossover sites in wild-type cells generally did not show recruitment of Msdla-GFP (96.2\% of 105 particles from 10 cells) and Wdr8-GFP (97.8\% of 91 particles from 10 cells) before the severing events (Fig. 6e, f and Supplementary Movies 29 and 30). Thus, Msd1 and Wdr8 do not play essential roles in either katanin recruitment or activity at microtubule crossovers.
Mutations in Msd1 or Wdr8 rescue interphase cell expansion phenotypes of katanin mutant plants. We examined the requirements of Msd1-Wdr8 and katanin on growth and cell shape in Arabidopsis plants. Growth of whole seedlings (Fig. 7a) and mature plants (Fig. $7 \mathrm{~b}$ ) was almost indistinguishable in wild type, $m s d 1 a m s d 1 b$, and $w d r 8$, but was severely inhibited in ktn1. Interestingly, the severe growth defects observed in $k t n 1$ were partially rescued by $m s d 1 a m s d 1 b$ or $w d r 8$. The growth rate of seedling primary roots was also highly impaired in ktn1, compared to wild type, msd1a msd1b, and $w d r 8$, and was also significantly (although not completely) rescued in msd1a msd1b 


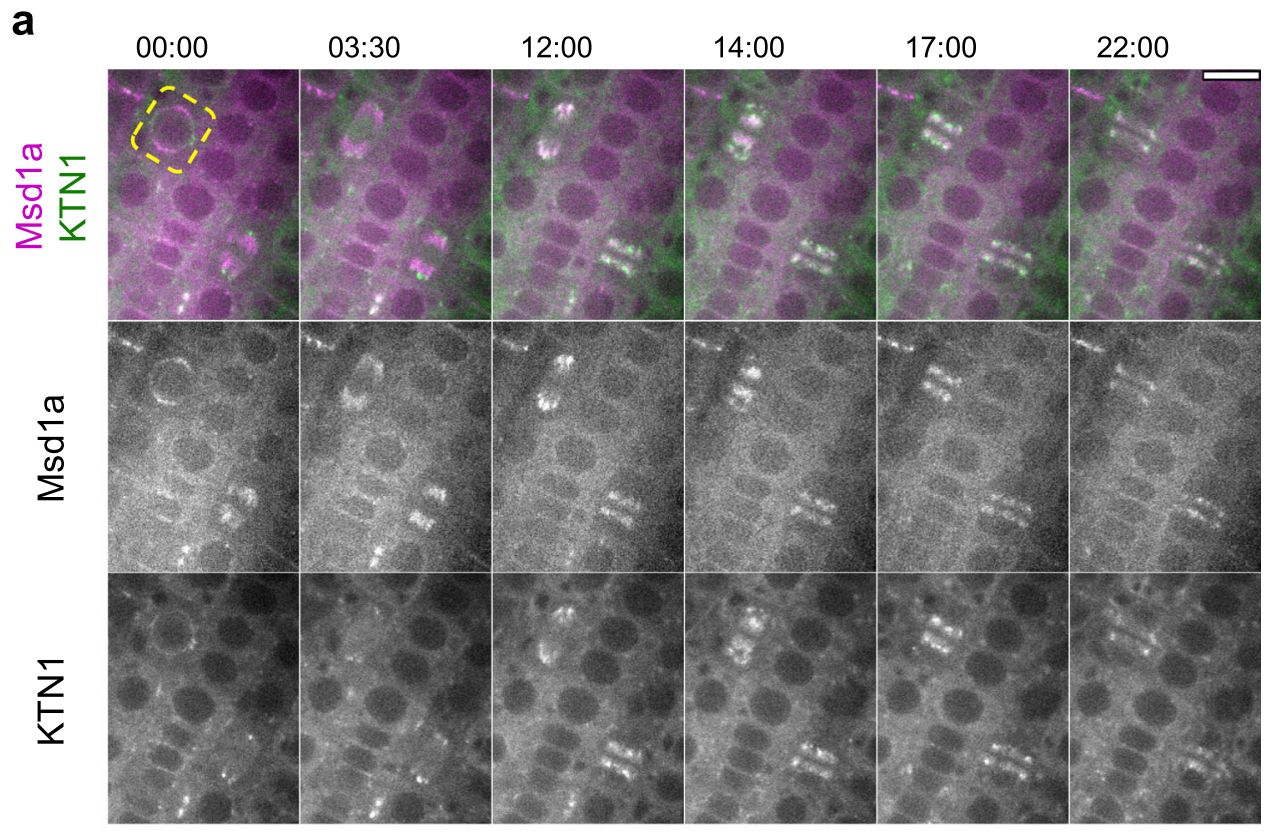

b
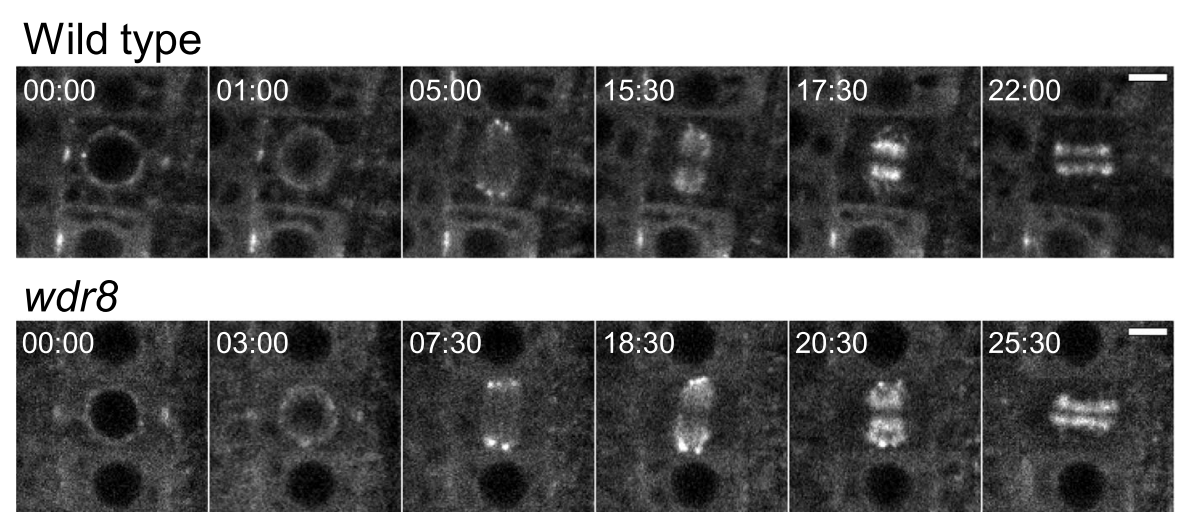

Fig. 5 Wdr8 is not required for katanin localization during cell division. a Colocalization of katanin and Msd1 during cell division. Time-lapse confocal microscopy images of GFP-KTN1 (green) and Msd1a-mCherry (magenta) in the dividing epidermal cells of the primary roots of 3-day-old wild-type seedlings. Beginning of the nuclear envelop breakdown in the root cell marked by the dotted yellow line sets the time zero points (minute : second). Katanin is substantially colocalized with Msd1 but accumulates more toward the minus ends of microtubules in spindles (12:00) and phragmoplasts (17:00). Four cytokinesis events from three plants were observed with similar results. Bar, $10 \mu \mathrm{m}$. b Subcellular localization of katanin during cell division. Time-lapse confocal microscopy images of GFP-KTN1 in the dividing epidermal cells of the primary roots of 3-day-old wild-type and wdr8 seedlings. Beginning of the nuclear envelop breakdown sets the time zero points (minute : second). Each images were averaged images of three $z$-slices taken with $0.7 \mu \mathrm{m}$ step. A total of ten and eight similar events were observed in wild-type cells (from eight plants) and wdr8 cells (from eight plants), respectively. Bars, $5 \mu \mathrm{m}$.

$k t n 1$ and $w d r 8 k t n 1$ (Fig. 7c). As root growth results from mitotic cell divisions in the meristem and subsequent rapid cell elongation during interphase, we investigated both phenotypes individually. In the ktn mutants, cytokinesis was severely impaired in the meristem as manifested by skewed cell division planes ${ }^{29}$. In the pericycle cell files of the primary roots, the katanin defect did not affect the mean angle between the transverse cell wall and the cell file's axis but significantly increased the variance of the orientation angles (Supplementary Fig. 8). The $m s d 1$ and $w d r 8$ mutations alone did not significantly affect cell division orientation and did not complement the cytokinetic defects of $k t n 1$. In interphase plant cells, cortical microtubule arrays are organized transverse to the longitudinal axis of axial organs and are required for anisotropic cell elongation, at least in part by promoting ordered cellulose deposition ${ }^{30,31}$. Confocal cross-sections of primary roots showed that cortical cells at the elongation zone and at the differentiation zone adopted long cylindrical shapes in wild type, $m s d 1 a m s d 1 b$, and $w d r 8$, but were radially swollen in $k t n 1$
(Fig. 7d). The isotropic cell expansion phenotype in $k t n 1$ was substantially rescued by genetic combination with msdla msd1b or $w d r 8$ (Fig. 7e). Taken together, these observations suggest that katanin-mediated severing antagonizes Msd1-Wdr8-mediated stabilization of interphase nucleation structures to allow for appropriate cortical microtubule array organization and postmitotic anisotropic expansion of plant cells.

\section{Discussion}

Our results show that in acentrosomal plant cells, the evolutionary conserved Msd1-Wdr8 complex anchors $\gamma$ TuRC to branching nucleation sites on interphase microtubules (Fig. 8). The absence of this complex does not affect the residence time of non-nucleating $\gamma$ TuRC complexes nor the time for $\gamma \mathrm{TuRC}$ complexes to nucleate following their arrival on the mother microtubule lattice; however, it decreases the percentages of branch-forming nucleation with respect to bundle-forming nucleation, indicating that the Msd1-Wdr8 complex specifically 
a

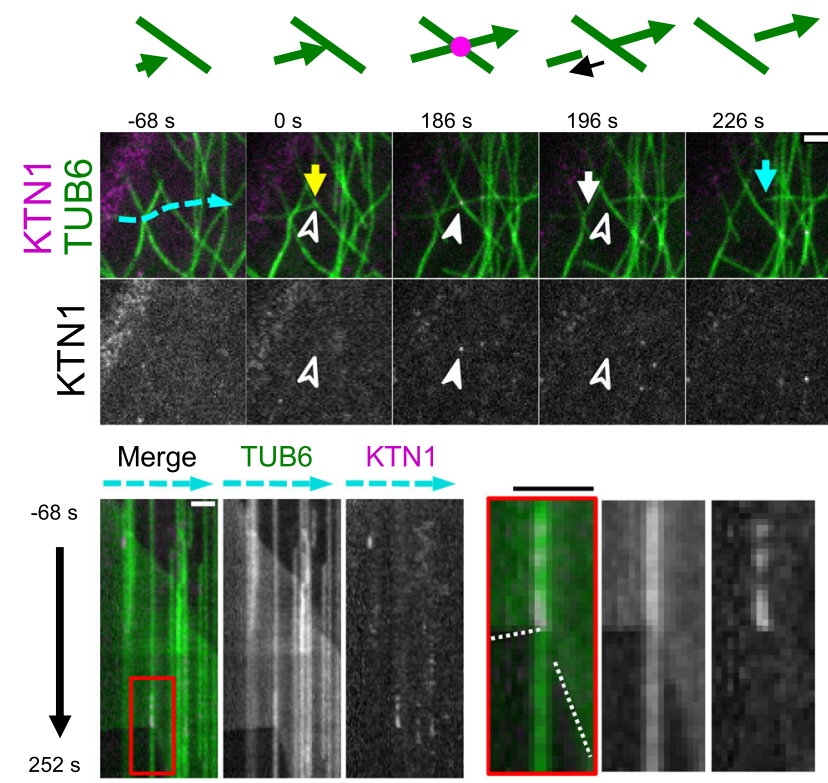

b $\quad$ dr8

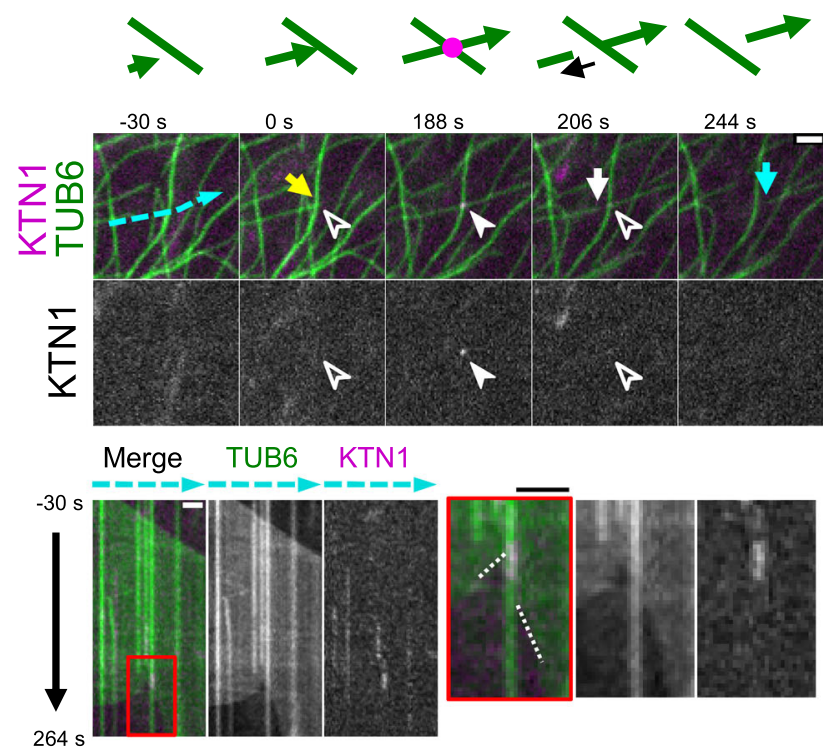

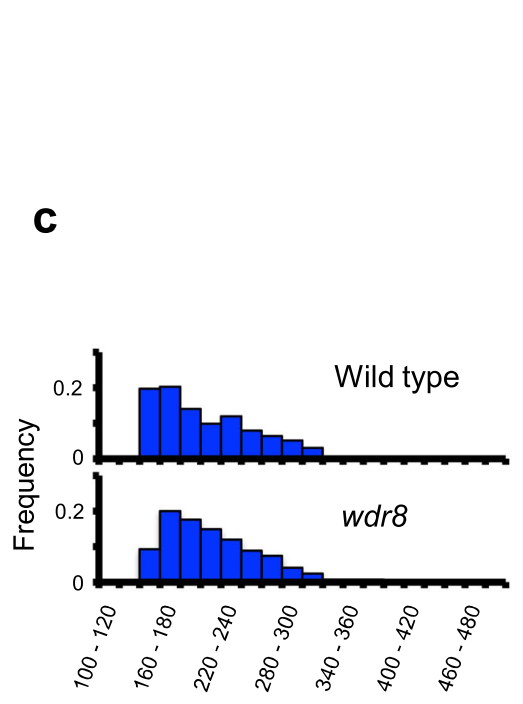

Max KTN1 intensity at ROI d

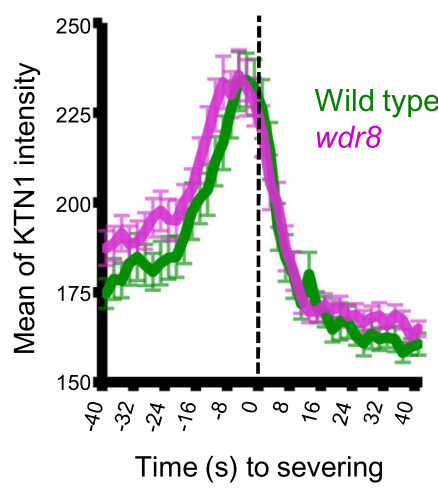

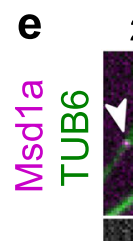

$\frac{\text { ர }}{\check{\delta}}$

$24 s$

$148 \mathrm{~s}$

$152 \mathrm{~s}$
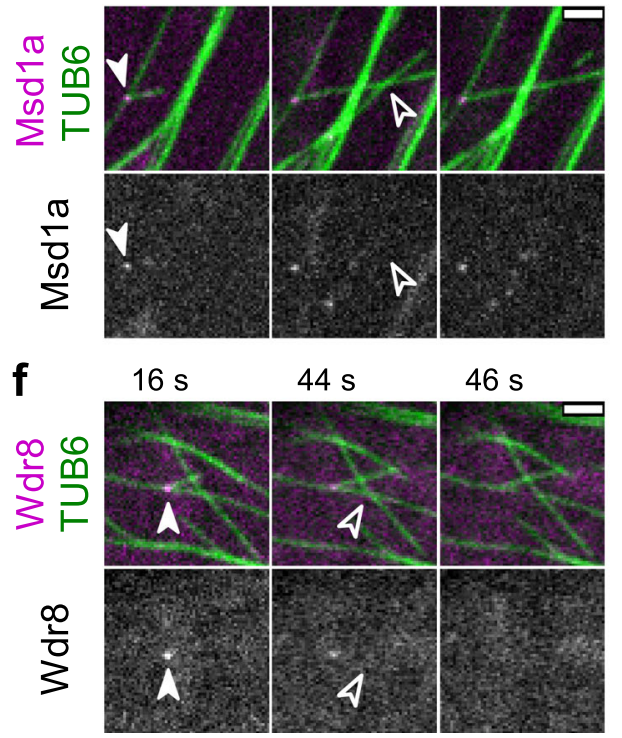

Fig. 6 Katanin recruitment to the microtubule crossover site does not require Msd1-Wdr8. a, b Time-lapse confocal microscopy images of katanin recruitment to the crossover sites in wild-type (a) and wdr8 (b) cells. Katanin (magenta) and microtubules (green) are labeled by GFP-KTN1 and mCherryTUB6, respectively. Open and closed arrowheads respectively indicate the absence and the presence of KTN1 particles. Yellow and white arrows show the growing and shrinking plus ends, whereas blue arrows indicate the shrinking minus ends of cortical microtubules. Kymographs track the dotted blue lines. Microtubule crossover events set the zero time points. Rectangle regions boxed by red lines are enlarged on the right where dotted white lines mark the plus $(+)$ and minus ( - ) ends of severed microtubules. A total of 54 and 65 similar events were observed in wild-type cells and wdr8 cells, respectively. Bars, $2 \mu \mathrm{m}$. c Distribution of GFP-KTN1 signal intensities at the crossover in wild type ( 720 ROls from 14 cells) and wdr8 ( 960 ROls from 11 cells). d Katanin accumulates at the crossover sites prior to severing in wild type (green; 42 events from 14 cells) and in wdr8 (magenta; 51 events from 11 cells).

Detachment of the severed microtubules were visually detected from the confocal images and was set to the time zero. The error bars indicate SD.

e, f Time-lapse confocal microscopy images of Msd1a-GFP (e) and Wdr8-GFP (f) (magenta), and mCherry-TUB6-labeled microtubules (green). Open and closed arrowheads respectively indicate the absence and the presence of Msd1/Wdr8 particles. Bars, $2 \mu \mathrm{m}$. e A daughter microtubule was formed at a branching nucleation site (labeled by Msd1a-GFP) and crossed bundled microtubules and another microtubule at $148 \mathrm{~s}$, which resulted in severing without prior recruitment of Msd1a (152 s). f A daughter microtubule was formed at a branching nucleation site (labeled by Wdr8-GFP) and crossed a microtubule at $16 \mathrm{~s}$, which resulted in severing in the absence of $\mathrm{Wdr} 8$ at $44 \mathrm{~s}$. Nucleation of the daughter microtubule is set to time zero.

stabilizes the $\gamma$ TuRC on the microtubule lattice once it has nucleated a branched daughter microtubule. Diffuse association of the Msd1 subunit with the microtubule lattice and the slight but significant time lag of the appearance of punctate Msd1 and Wdr8 at $\gamma$ TuRC-labeled cortical nucleation sites suggest that the complex either specifically assembles and accumulates at these locations or that the complexes are assembled on the lattice in general and then transported to the lattice-bound $\gamma$ TuRC. In fission yeast, a minus-end directed kinesin $\mathrm{Pkll}$ is required to transport the Msd1-Wdr8 complex along the spindle 

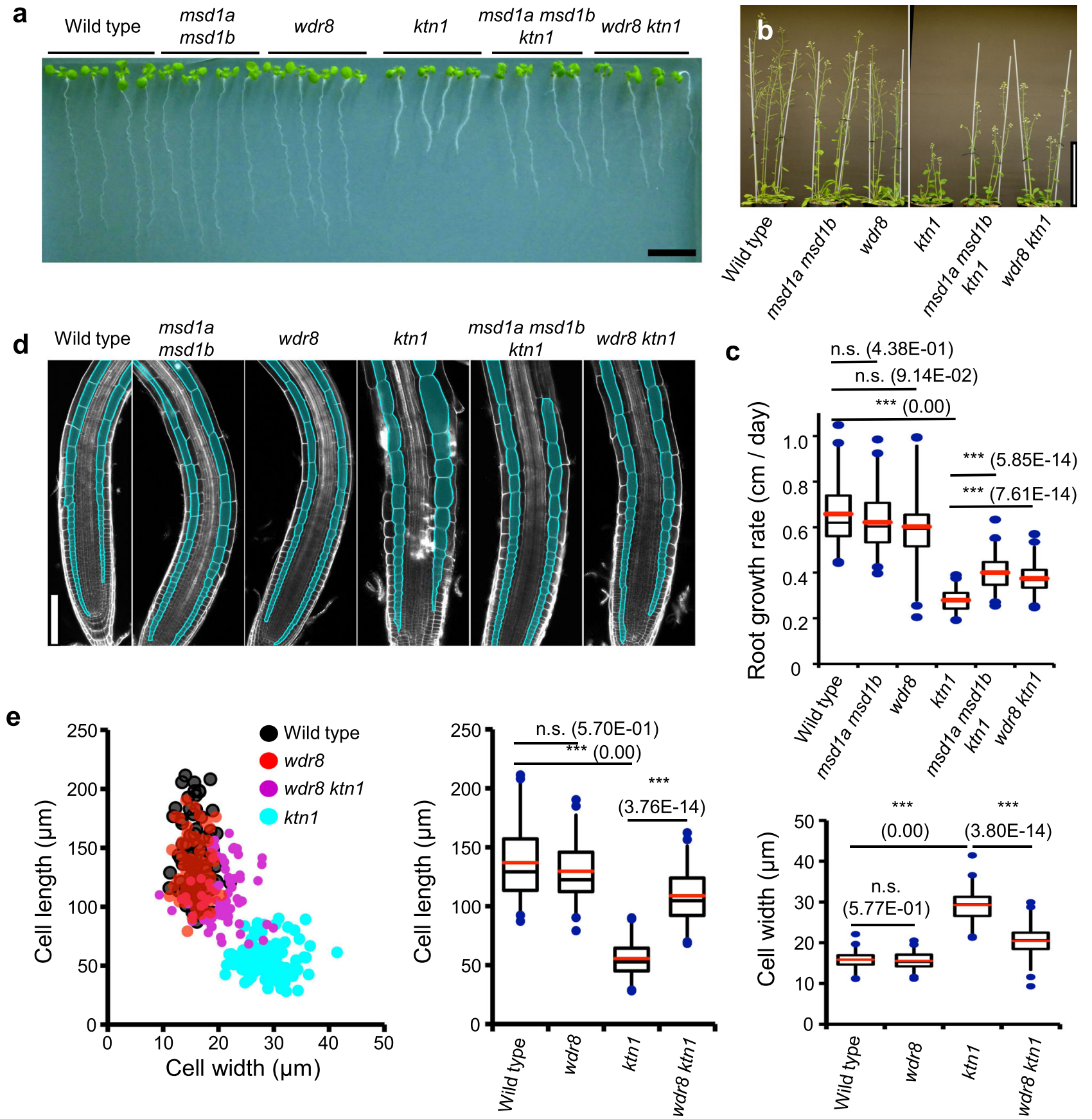

Fig. 7 Mutations in the Msd1-Wdr8 complex complement the cell shape defects of katanin mutants. a Eight-day-old Arabidopsis seedlings grown on a vertically placed agar medium. Bar, $10 \mathrm{~mm}$. b Growth and statute of flowering Arabidopsis plants. Bar, $10 \mathrm{~cm}$. c Growth rate (cm per day) measured by daily increment in the length of primary roots from 5 to 10 days after germination in wild type (20 seedlings), msd1a msd1b (20 seedlings), wdr8 (20 seedlings), ktn1 (19 seedlings), msd1a msd1b ktn1 (18 seedlings), and wdr8 ktn1 (18 seedlings). The bottom and top of each box represent the first and third quartiles, respectively; the horizontal line inside each box is the median (second quartile) and the red horizontal line represents the average. The whiskers range between 2 nd percentile and 98th percentile. n.s., not significant and ${ }^{{ }^{* \star}} p<0.0001$, determined by the Steel-Dwass test. Exact $p$-values are indicated in parentheses. d Root tip morphology of indicated genotypes. Cortex cell files are highlighted with the turquoise paint. Bar, $100 \mu \mathrm{m}$. e Cortex cell shape of $4-$ day-old primary roots at the differentiation zone where cell expansion has ceased in wild type (98 cells from 4 seedlings), wdr8 (47 cells from 3 seedlings), ktn1 (94 cells from 2 seedlings), and wdr8 ktn1 (76 cells from 2 seedlings). Left, cell length and cell width of individual cells. Middle, cell length. Right, cell width. n.s., not significant and ${ }^{\star \star \star} p<0.0001$, determined by the Steel-Dwass test. Exact $p$-values are indicated in parentheses. The elements of the box plot are defined as in $\mathbf{c}$.

microtubule to the spindle-pole body ${ }^{32}$. Arabidopsis microtubule motors might function in a similar manner.

How katanin is recruited to specific cellular addresses to sever microtubules is poorly understood. In a previous study, purified katanin complexes of Caenorhabditis elegans preferentially sever at intersections between two microtubules in vitro ${ }^{33}$, suggesting that specific recruitment factors may not always be necessary. Here we have shown that Msd1-Wdr8 not only functions to stabilize branching nucleation structures in cortical arrays of Arabidopsis cells, but that it also functions as an essential factor to 


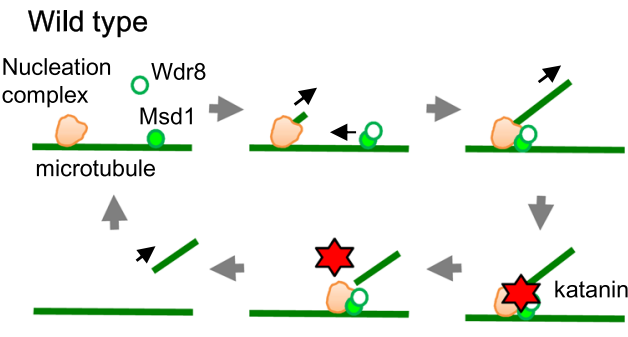

katanin

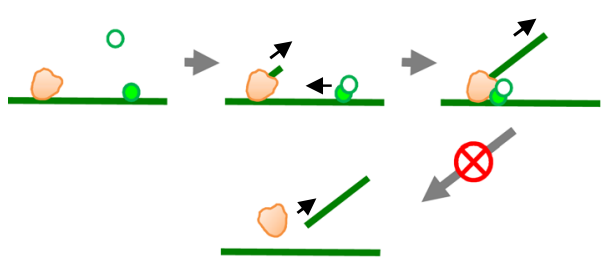

msd1a msd1b, wdr8 and wdr8 katanin

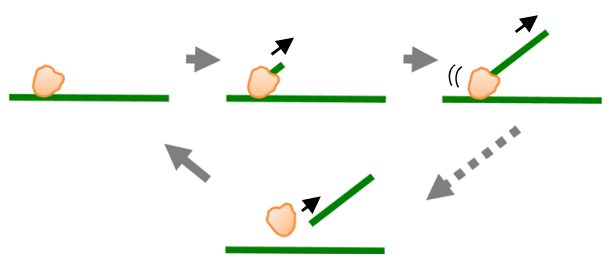

Fig. 8 Model for stabilization of branching nucleation sites and recruitment of katanin by the Msd1-Wdr8 complex. In wild-type cells, lattice-bound Msd1 (filled green circle) recruits cytoplasmic Wdr8 (open green circle) to form a heteromeric complex, which is translocated to and associated with $\gamma$ TuRC (orange) on a preexisting microtubule (green line). After nucleation of a microtubule, katanin is recruited by Msd1-Wdr8 and severs the minus end of the daughter microtubule. Direct physical interactions between katanin and Msd1-Wdr8 are yet to be demonstrated. In msd1 and wdr8 cells, nucleated $\gamma$ TuRC is not stably tethered to the mother microtubule and is eventually dissociated in the absence of the katanin activity. The wdr8 mutation (and probably the msd1 mutation as well) is epistatic to the katanin mutation. The figure was prepared originally by the authors.

recruit katanin to these sites for their disassembly. Such counteracting dual functions on the nucleation site stability likely underlie apparently normal growth of $m s d 1$ and $w d r 8$ mutant plants under standard growth conditions. An analogous stabilization-destabilization mechanism of microtubule organization has been reported for mammalian microtubule minus-endregulating proteins CAMSAP1 and CAMSAP2, which form stretches of stabilized microtubule lattice at the minus ends, and recruit katanin to antagonize the formation of long CAMSAPdecorated stretches ${ }^{34}$.

The plant Msd1-Wdr8 complex would endow plant cells with the ability to place release of daughter microtubules under the strict regulation of the katanin activity. Spindle length in Xenopus species is controlled by inhibitory phosphorylation on the katanin p60 subunit ${ }^{35}$. Strict dependency of microtubule severing on katanin activity may make remodeling of microtubule array organization adaptable to changing cellular status and environmental stimuli. Although it is not well understood what developmental and environmental cues control the katanin activities in plant cells, the $m s d 1$ and $w d r 8$ mutants, in which daughter microtubules are strictly released by the katanin-independent manner, may be useful for exploring environmentally stimulated microtubule array remodeling processes in which katanin activation plays a critical role.

\begin{abstract}
Methods
Plant materials and growth conditions. A. thaliana ecotype Columbia- 0 was used and the original plants and their transgenic derivatives expressing marker proteins were all referred to as the wild type in this study. Seedlings were grown on $1.5 \%$ agar medium containing a half-strength Murashige and Skoog salt mixture $(\mathrm{pH}$ 5.7) and $1 \%$ sucrose at $22{ }^{\circ} \mathrm{C}$ under a long-day photoperiod $(16 \mathrm{~h}$ light $/ 8 \mathrm{~h}$ dark) condition or in the dark. T-DNA tagged lines of Msd1a (msd1a-1, SALK_148799; $m s d 1 a-2, \mathrm{GABI} 5557 \mathrm{~F} 06), M s d 1 b$ (msd1b-1, SALK_132426; $m s d 1 b-2$,

GABI_596B10), and Wdr8 ( $w d r 8$-1, SALK_093768) were obtained from the Arabidopsis Biological Resource Center (https://abrc.osu.edu/). To confirm the T-DNA insertion sites (Supplementary Fig. 2), genomic regions flanking the insertion sites were amplified by PCR using the primer sets listed in Supplementary Table S1 and were sequenced. The $k t n 1-2$ allele was reported previously ${ }^{36}$.
\end{abstract}

Transgenic plant lines expressing marker proteins. The Msdla-GFP, Msd1bGFP, Wdr8-GFP, and Msdla-mCherry constructs were generated by amplifying the corresponding genomic fragments of Msd1a (At5g57410; $7157 \mathrm{bp}$ ), Msd1b (At2g18876; $6952 \mathrm{bp}$ ), and Wdr8 (At5g07590; $5797 \mathrm{bp}$ ) with the primers listed in Supplementary Table 1. GFP and mCherry were fused to the gene of interest separated by a GGGGSGGGGSGGGGS-linker ${ }^{37}$, just before the stop codon. These fluorescent protein-fusion constructs were transferred to $\mathrm{pBIN} 40^{38}$ (with a hygromycin-resistance gene) or pBIN $30^{39}$ (with a bialaphos-resistance gene), which had been modified from pBIN19. An Agrobacterium-mediated transformation method ${ }^{40}$ was used to generate transgenic plants. The transgenic plant lines expressing the fusion proteins were crossed with a microtubule-marker line expressing mCherry-TUB6 ${ }^{41}$ for co-visualization with microtubules. An MsdlamCherry transgenic plant was crossed with an MZT1a-GFP line ${ }^{42}$ for colocalization analysis. A list of primers used for molecular cloning is provided in Supplementary Table 1 .

To facilitate generation of dual marker lines in various mutant backgrounds, we constructed a microtubule nucleation marker binary vector, which harbors both an mCherry-TUB6 cassette for microtubule labeling and an MZT1a-GFP cassette for $\gamma$ TuRC visualization. After a StuI cloning site was introduced adjacent to the multi-cloning site of pBIN40, the UBI10 promoter-driven mCherry-TUB6-expressing cassette ${ }^{41}$ was cloned into the StuI site and the MZT1a-GFP-expressing cassette ${ }^{42}$ was then cloned into the AscI site of the resulting pBIN4100 plasmid. This binary vector was used to transform wild-type Arabidopsis plants to establish a microtubule nucleation dual marker line, which was then crossed to mutant plants. Marker lines with homozygous mutations for msd1a-1 msd1b-1, wdr8-1 or ktn1-2 were identified in subsequent generations. To co-visualize katanin and microtubules, the GFP-KTN1-expressing plant in the $k t n 1-2$ background ${ }^{43}$ and the mCherry-TUB6 line were independently crossed with $w d r 8-1$, and the progeny lines harboring both constructs in the ktn1-2 and ktn1-2 wdr8-1 homozygous backgrounds were identified in subsequent generations.

Immunopurification and mass spectrometry. Methods to purify GFP-tagged proteins and associated proteins from Arabidopsis plant extracts were described previously ${ }^{36,42}$. In brief, crude protein extracts in the extraction buffer $(50 \mathrm{mM}$ HEPES, $150 \mathrm{mM} \mathrm{NaCl}, 1 \% \mathrm{NP}-40,2 \mathrm{mM}$ phenylmethylsulfonyl fluoride, Complete Protease Inhibitor EDTA-free cocktail (Roche), $\mathrm{pH}$ 7.5) were prepared from 10- or 12-day-old light-grown transgenic plants expressing a target GFP-fused protein. Immunoprecipitation was carried out using the $\mu \mathrm{MACS}$ GFP-tag protein purification kit (\#130-091-125; Miltenyi Biotec, Bergisch Gladbanch, Germany), basically by following the manufacturer's protocol, except that the above extraction buffer was used in the first washing step. This kit includes $\mu$ MACS Microbeads that are conjugated to an anti-GFP monoclonal antibody. Purified proteins were separated by SDS-polyacrylamide gel electrophoresis, stained with SYPRO Rubby (Thermo Fisher), and pooled into several fractions corresponding to their molecular weight. In-gel protein digestion to peptides and subsequent LC-MS/MS analysis was performed as reported ${ }^{36}$.

Time-lapse imaging. Dynamics of Msd1, Wdr8, MZT1, and KTN1 with respect to locations of cortical microtubules in interphase cells was analyzed in adaxial pavement cells of 13- to 15-day-old cotyledons. Excised cotyledons were mounted on slide glasses with shallow holes (TOSHINRIKO CO., LTD) and cover glasses were immobilized on slide glasses with commercial nail polish to minimize drifting of specimens during observation. Images in Figs. 1-3 were obtained using a confocal microscope system previously reported ${ }^{44}$. mCherry and GFP were sequentially excited with 100 and $200 \mathrm{~ms}$ exposure, respectively, and images from three consecutive excitations were averaged. Images were acquired every $2 \mathrm{~s}$ for 5-15 min. For Fig. 4, an Olympus IX-83 inverted microscope equipped with a spinning disk confocal unit Yokogawa CSU-W1, a dichroic mirror DM405/488/561, an Andor EM-CCD camera iXron3 888, and a $\times 100,1.40$ numerical aperture (NA) UPlanSApo oil-immersion objective (Olympus) were used. mCherry and GFP were sequentially excited by 561 and $488 \mathrm{~nm}$ OPSL lasers (Coherent) for 400 and 800 
$\mathrm{ms}$, and images were collected every $2.5 \mathrm{~s}$ for $10 \mathrm{~min}$ with $617 / 73$ and $520 / 35$ emission filters, respectively.

Colocalization of Msd1a-mCherry and MZT1a-GFP (Fig. 1f) was analyzed in hypocotyl epidermal cells of 3-day-old etiolated seedlings with a confocal microscope system reported previously ${ }^{36}$. Specimens were prepared by the agar pads method as previously reported ${ }^{45}$. Both GFP and mCherry were excited with $800 \mathrm{~ms}$ exposures, and images were taken with $2.5 \mathrm{~s}$ intervals for $15 \mathrm{~min}$. Two experiments with reverse orders of excitations were performed and the data were pooled.

Time-lapse imaging of mitotic localization of Msd1 and KTN1 in root tip cells (Fig. 5a) was done in glass-based dishes (IWAKI, \#3910-035). Four-day-old seedlings were mounted on dishes with nutrition-containing agar block. A previously reported microscope system ${ }^{44}$ was used with a $\times 60,1.49 \mathrm{NA} \mathrm{CFI}$ Apochromat TIRF oil-immersion objective. mCherry and GFP were excited in this order with 200 and $150 \mathrm{~ms}$ exposure. Images were taken with $30 \mathrm{~s}$ intervals. Mitotic KTN1 localization in wild-type and $w d r 8$ mutant backgrounds (Fig. 5b) used the same microscope system as Fig. 4, with a $\times 60,1.30$ NA UPlanSApo siliconimmersion objective. Images were taken as $0.5 \mu \mathrm{m}$ step $z$-stacks and with $30 \mathrm{~s}$ intervals.

Image processing and analysis. Image processing and analyses were performed with the Fiji/ImageJ software. At the beginning of image analysis, image drifting was corrected using microtubule channels with an ImageJ plugin StackReg ${ }^{46}$. To reduce background signals of each images, custom Walking Average macro and the ImageJ subtract background tool were used.

In Figs. 1 and 2, GFP-labeled particles of Msd1, Wdr8, and MZT1a were first picked up and microtubule dynamics in the region of interest were then analyzed in the mCherry channel. To measure the time required to release $\gamma \mathrm{TuRC}$ from microtubules (Fig. 2g), those MZT1a particles which appeared on the cortical microtubules and later disappeared after branch-forming nucleation during the image capture period (up to $600 \mathrm{~s}$ ) were analyzed.

The dynamics of mCherry-TUB6-labeled microtubules was used to identify the nucleation events in which both initiation and release of daughter microtubules were visible (Fig. 3), and the crossover events in which both crossover formation and subsequent severing of overlaid microtubules were visible (Fig. 6). The signal intensities of GFP-KTN1 at the severed regions ( 5 pixel $\times 5$ pixel; $0.52 \mu \mathrm{m}^{2}$ ) were recorded over time.

Arrival to and disappearance from the cell cortex of Msdla-mCherry and MZT1a-GFP were monitored by using Fiji/ImageJ plugin TrackMate ${ }^{47}$ with the LoG detector filter and the detection spot radius size of 2.5 pixel $(0.26 \mu \mathrm{m})$. Fluorescent particles were tracked by using the nearest-neighbor search filter with a maximal distance allowance setting of 5 pixel. The time lag $(800 \mathrm{~ms})$ required for the sequential excitations was corrected.

Yeast two-hybrid interaction assay. Open reading frames for $M s d 1 a, M s d 1 b$, and $W d r 8$ cDNAs were obtained after reverse-transcription PCR from seedling RNA using primer sets in Supplementary Table S1, followed by cloning of the PCR fragments into pHSG399. The cDNAs were excised by BamHI and were inserted to the BamHI site in pGADT7 (Clontech) and pGBKT7 (Clontech) for translational fusions to the Gal4 activation domain and to the Gal4-binging domain, respectively. Other plasmids and experimental procedures have been previously reported ${ }^{42}$.

Phenotypic analysis. Primary roots of Arabidopsis seedlings grown on vertically placed agar plates were photographed every day after 5-10 days of germination with a conventional image scanner (EPSON GT-S650) and the root length was analyzed by a FIJI/ImageJ software. To analyze cell shapes, primary roots of lightgrown 4-day-old seedlings were stained with propidium iodide at $10 \mu \mathrm{g} \mathrm{mL}^{-1}$ and observed with a confocal microscope Leica SP8 with a $\times 20,0.75$ NA HC PL APO CS2 objective (Leica). To analyze root cortex cells of the differentiation region, light-grown 4-day-old seedlings were fixed with $4 \%$ formaldehyde for $1 \mathrm{~h}$, followed by several washes with the $1 \times$ phosphate-buffered saline, and then stained with a 1: 5 diluted solution of Calcoflour White Stain (Sigma-Aldrich, \#18909) for $30 \mathrm{~min}$. After washing out an excess staining solution, root samples were mounted with the ClearSee solution for deep tissue imaging ${ }^{48}$. Images were taken as $0.7 \mu \mathrm{m}$-step $z$ stacks with a Zeiss inverted microscope Axio Observer equipped with a confocal system LSM800 and a $\times 40,1.1 \mathrm{NA}$ LD C-Apochromat water-immersion objective (Zeiss). The root differentiation zone was determined by the proto-xylem differentiation

Statistical information. Statistical analyses were performed using Microsoft Excel plugin software, StatPlus (AnalystSoft), Prism8 (GraphPad Software), and R software ${ }^{49}$. The data are presented as mean \pm SD and $n$-values are shown in the figure legends. For boxplots, the bottom and top of each box represent the first and third quartiles, respectively. The horizontal line inside each box is the median (second quartile) and the red horizontal line represents the average. The whiskers range between 2 nd percentile and 98 th percentile. Data points outside this range are considered as outliers and are indicated as circles. n.s. indicates not significant,
${ }^{*} p<0.01,{ }^{* *} p<0.001,{ }^{* * *} p<0.0001$. To compare means for multiple groups, Steel-Dwass test was performed using $\mathrm{R}$ software.

Reporting summary. Further information on research design is available in the Nature Research Reporting Summary linked to this article.

\section{Data availability}

The mass spectrometry proteomics data have been deposited to the ProteomeXchange Consortium via the PRIDE [http://www.ebi.ac.uk/pride] partner repository with the dataset identifier PXD026063 and 10.6019/PXD026063. Other datasets generated during the current study are available from the corresponding authors on request. Source data are provided with this paper.

Received: 4 June 2020; Accepted: 25 May 2021; Published online: 17 June 2021

\section{References}

1. Hashimoto, T. Dynamics and regulation of plant interphase microtubules: a comparative view. Curr. Opin. Plant Biol. 6, 568-576 (2003).

2. McNally, F. J. \& Roll-Mecak, A. Microtubule-severing enzymes: from cellular functions to molecular mechanism. J. Cell Biol. 217, 4057-4069 (2019).

3. Nakamura, M. Microtubule nucleating and severing enzymes for modifying microtubule array organization and cell morphogenesis in response to environmental cues. N. Phytol. 205, 1022-1027 (2015).

4. Toya, M. et al. $\gamma$-Tubulin complex-mediated anchoring of spindle microtubules to spindle-pole bodies requires Msd1 in fission yeast. Nat. Cell Biol. 9, 646-653 (2007).

5. Bärenz, F. et al. The centriolar satellite protein SSX2IP promotes centrosome maturation. J. Cell Biol. 202, 81-95 (2013).

6. Hori, A., Ikebe, C., Tada, M. \& Toda, T. Msd1/SSX2IP-dependent microtubule anchorage ensures orientation and primary cilia formation. EMBO Rep. 15, 175-184 (2014).

7. Hori, A., Peddie, C. J., Collinson, L. M. \& Toda, T. Centriolar satellite- and hMsd1/SSX2IP-dependent microtubule anchoring is critical for centriole assembly. Mol. Biol. Cell 26, 2005-2019 (2015).

8. Shen, K.-F. \& Osmani, S. A. Regulation of mitosis by the NIMA kinase involves TINA and its newly discovered partner, An-WDR8, at spindle pole bodies. Mol. Biol. Cell 24, 3842-3856 (2013).

9. Hori, A. et al. The conserved Wdr8-hMsd1/SSX2IP complex localises to the centrosome and ensures proper spindle length and orientation. Biochem. Biophys. Res. Commun. 468, 39-45 (2015).

10. Kurtulmus, B. et al. WDR8 is a centriolar satellite and centriole-associated protein that promotes ciliary vesicle docking during ciliogenesis. J. Cell Sci. 129, 621-636 (2016).

11. Inoue, D. et al. Expression of the novel maternal centrosome assembly factor Wdr8 is required for vertebrate embryonic mitoses. Nat. Commun. 8, 14090 (2017).

12. Murata, T. et al. Microtubule-dependent microtubule nucleation based on recruitment of $\gamma$-tubulin in higher plants. Nat. Cell Biol. 7, 961-968 (2005).

13. Chan, J., Sambade, A., Calder, G. \& Lloyd, C. Arabidopsis cortical microtubules are initiated along, as well as branching from, existing microtubules. Plant Cell 21, 2298-2306 (2009).

14. Nakamura, M., Ehrhardt, D. W. \& Hashimoto, T. Microtubule and katanindependent dynamics of microtubule nucleation complexes in the acentrosomal Arabidopsis cortical array. Nat. Cell Biol. 12, 1064-1070 (2010).

15. Nakamura, M. \& Hashimoto, T. A mutation in the Arabidopsis $\gamma$-tubulincontaining complex subunit causes helical growth and abnormal microtubule branching. J. Cell Sci. 122, 2208-2217 (2009).

16. Wightman, R. \& Turner, S. R. Severing at sites of microtubule crossover contributes to microtubule alignment in cortical arrays. Plant J. 52, 742-751 (2007).

17. Zhang, Q., Fishel, E., Bertroche, T. \& Dixit, R. Microtubule severing at crossover sites by katanin generates ordered cortical microtubule arrays in Arabidopsis. Curr. Biol. 23, 2191-2195 (2013).

18. Lindeboom, J. J. et al. A mechanism for reorientation of cortical microtubule arrays driven by microtubule severing. Science 342, 1245533 (2013)

19. Wang, C. et al. KTN80 confers precision to microtubule severing by specific targeting of katanin complexes in plant cells. EMBO J. 36, 3435-3447 (2017)

20. Murata, T. et al. Mechanism of microtubule array expansion in the cytokinetic phragmoplast. Nat. Commun. 4, 1967 (2013).

21. Panteris, E., Adamakis, I.-D. S., Voulgari, G. \& Papadopoulou, G. A role of katanin in plant cell division: microtubule organization in dividing root cells of fra2 and lue1 Arabidopsis thaliana mutants. Cytoskeleton 68, 401-413 (2011). 
22. Komis, G. et al. Katanin effects on dynamics of cortical microtubules and mitotic arrays in Arabidopsis thaliana revealed by advanced live-cell imaging. Front. Plant Sci. 8, 866 (2017).

23. Hamada, T. et al. Purification and characterization of novel microtubuleassociated proteins from Arabidopsis cell suspension cultures. Plant Physiol. 163, 1804-1816 (2013).

24. Shaw, S., Kamyar, R. \& Ehrhardt, D. W. Sustained microtubule treadmilling in Arabidopsis cortical arrays. Science 300, 1715-1718 (2003).

25. Nakamura, M. et al. Arabidopsis GCP3-INTERACTING PROTEIN 1/ MOZART1 is an integral component of the $\gamma$-tubulin-containing microtubule nucleating complex. Plant J. 71, 216-225 (2012).

26. Pastuglia, M. et al. $\gamma$-Tubulin is essential for microtubule organization and development in Arabidopsis. Plant Cell 18, 1412-1425 (2006).

27. Ho, C.-M. K. et al. Augmin plays a critical role in organizing the spindle and phragmoplast microtubule arrays in Arabidopsis. Plant Cell 23, 2606-2618 (2011).

28. Sasaki, T. et al. A novel katanin-tethering machinery accelerates cytokinesis. Curr. Biol. 29, 4060-4070 (2019).

29. Bichet, A. et al. BOTERO1 is required for normal orientation of cortical microtubules and anisotropic cell expansion in Arabidopsis. Plant J. 25, 137-148 (2001).

30. Ehrhardt, D. W. \& Shaw, S. L. Microtubule dynamics and organization of the plant cortical array. Annu. Rev. Plant Biol. 57, 859-875 (2006).

31. Paredez, A. R., Somerville, C. R. \& Ehrhardt, D. W. Visualization of cellulose synthase demonstrates functional association with microtubules. Science 312, 1491-1495 (2006).

32. Yukawa, M., Ikebe, C. \& Toda, T. The Msd1-Wdr8-Pkl1 complex anchors microtubule minus ends to fission yeast spindle pole bodies. J. Cell Biol. 209, 549-562 (2015).

33. McNally, K. et al. Katanin maintains meiotic metaphase chromosome alignment and spindle structure in vivo and has multiple effects on microtubules in vitro. Mol. Biol. Cell 25, 1037-1049 (2014).

34. Jiang, K. et al. Microtubule minus-end stabilization by polymerization-driven CAMSAP deposition. Dev. Cell 28, 295-309 (2014).

35. Loughlin, R. et al. Katanin contributes to interspecies spindle length scaling in Xenopus. Cell 147, 1397-1407 (2011).

36. Nakamura, M., Ehrhardt, D. W. \& Hashimoto, T. Microtubule and katanindependent dynamics of microtubule nucleation complexes in the acentrosomal Arabidopsis cortical array. Nat. Cell Biol. 12, 1064-1070 (2010).

37. Shimozono, S. \& Miyawaki, A. Engineering FRET constructs using CFP and YFP. Methods Cell Biol. 85, 381-393 (2008).

38. Hirota, A., Kato, T., Fukaki, H., Aida, M. \& Tasaka, M. The auxin-regulated AP2/EREBP gene PUCHI is required for morphogenesis in the early lateral root primordium of Arabidopsis. Plant Cell 19, 2156-2168 (2007).

39. Miyashima, S., Koi, S., Hashimoto, T. \& Nakajima, K. Non-cell-autonomous microRNA165 acts in a dose-dependent manner to regulate multiple differentiation status in the Arabidopsis root. Development 138, 2303-2313 (2011).

40. Clough, S. J. \& Bent, A. F. Floral dip: a simplified method for Agrobacteriummediated transformation of Arabidopsis thaliana. Plant J. 16, 735-743 (1998).

41. Fujita, S. et al. An atypical tubulin kinase mediates stress-induced microtubule depolymerization in Arabidopsis. Curr. Biol. 23, 1969-1978 (2013).

42. Nakamura, M. et al. Arabidopsis GCP3-interacting protein 1/MOZART 1 is an integral component of the $\gamma$-tubulin-containing microtubule nucleating complex. Plant J. 71, 216-225 (2012).

43. Lindeboom, J. J. et al. A mechanism for reorientation of cortical microtubule arrays driven by microtubule severing. Science 342, 1245533 (2013).

44. Wong, J. H. \& Hashimoto, T. Novel Arabidopsis microtubule-associated proteins track growing microtubule plus ends. BMC Plant Biol. 17, 1-11 (2017).

45. Gutierrez, R., Lindeboom, J., Paredez, A., Emons, A. \& Ehrhardt, D. Arabidopsis cortical microtubules position cellulose synthase delivery to the plasma membrane and interact with cellulose synthase trafficking compartments. Nat. Cell Biol. 11, 797-806 (2009).

46. Thévenaz, P., Ruttimann, U. E. \& Unser, M. A pyramid approach to subpixel registration based on intensity. IEEE Trans. Image Process. 7, 27-41 (1998).

47. Tinevez, J. Y. et al. TrackMate: an open and extensible platform for singleparticle tracking. Methods 115, 80-90 (2017).

48. Kurihara, D., Mizuta, Y., Sato, Y. \& Higashiyama, T. ClearSee: a rapid optical clearing reagent for whole-plant fluorescence imaging. Development 142, 4168-4179 (2015)

49. Ihaka, R. \& Gentleman., R. R: a language for data analysis and graphics. J. Comp. Graph. Stat. 5, 299-314 (1996).

\section{Acknowledgements}

We thank M. Kawaguchi for the help in the yeast two-hybrid assays and Y. Sakumura for discussion on data processing. This work was supported by Grant-in-Aid for Scientific Research (B) to T.H. (17H03698) and S.M. (15K21750, 15H05962, and 15H05955), and Human Frontier Science Program Grant to M.N. (18KK0195) from Japan Society for the Promotion of Science (JSPS), Japan, and NSF Grant (1158372) to D.W.E. ITbM was supported by World Premier International Research Center Initiative (WPI), Japan.

\section{Author contributions}

T.H. and M.N. conceived the project. N.Y., M.N. and T.K. performed the experiments T.H., M.N., N.Y. and D.W.E. analyzed the data and wrote the manuscript. All authors discussed the results and commented on the manuscript.

\section{Competing interests}

The authors declare no competing interests.

\section{Additional information}

Supplementary information The online version contains supplementary material available at https://doi.org/10.1038/s41467-021-24067-y.

Correspondence and requests for materials should be addressed to M.N. or T.H.

Peer review information Nature Communications thanks Bo Liu and the other, anonymous reviewer(s) for their contribution to the peer review of this work. Peer review reports are available.

Reprints and permission information is available at http://www.nature.com/reprints

Publisher's note Springer Nature remains neutral with regard to jurisdictional claims in published maps and institutional affiliations.

(c) (i) Open Access This article is licensed under a Creative Commons c. Attribution 4.0 International License, which permits use, sharing, adaptation, distribution and reproduction in any medium or format, as long as you give appropriate credit to the original author(s) and the source, provide a link to the Creative Commons license, and indicate if changes were made. The images or other third party material in this article are included in the article's Creative Commons license, unless indicated otherwise in a credit line to the material. If material is not included in the article's Creative Commons license and your intended use is not permitted by statutory regulation or exceeds the permitted use, you will need to obtain permission directly from the copyright holder. To view a copy of this license, visit http://creativecommons.org/ licenses/by/4.0/

(C) The Author(s) 2021 